\title{
How bound water regulates wood drying
}

Hélène Penvern ${ }^{1}$, Meng Zhou ${ }^{1}$, Benjamin Maillet ${ }^{1}$, Denis Courtier-Murias ${ }^{1,2}$, Mario Scheel ${ }^{3}$, Jonathan Perrin ${ }^{3}$, Timm Weitkamp ${ }^{3}$, Sandrine Bardet ${ }^{4}$, Sabine Caré ${ }^{1}$, Philippe Coussot ${ }^{1}$

${ }^{1}$ Lab. Navier, Ecole des Ponts, Univ Gustave Eiffel, CNRS, 77420 Marne la Vallée, France

${ }^{2}$ GERS-LEE, Univ Gustave Eiffel, IFSTTAR, F-44344 Bouguenais, France

${ }^{3}$ Synchrotron SOLEIL, 91192 Gif-sur-Yvette, France

${ }^{4}$ LMGC, Univ. Montpellier, CNRS, 34090 Montpellier, France

* Corresponding author: Philippe Coussot (philippe.coussot@univ-eiffel.fr)

\begin{abstract}
For most wood-based product uses it is essential to remove a large part of the water content from wet or green (fresh-cut) wood, to reduce further dimensional variations under varying humidity conditions, improve its mechanical characteristics and protect it from biological attacks. However, the internal mechanisms of drying are not fully described. Here we observe drying at different scales using macroscopic measurements (weighing), NMR (Nuclear Magnetic Resonance) measurements allowing to distinguish bound and free water contents, and XRCT (X-ray computed tomography) images of air-liquid interfaces at the smallest pore scale (wood lumens). We show that during wood drying, even well above the Fiber Saturation Point, bound water diffusion in cell walls (instead of capillary effects) ensures the extraction of liquid water from pores and its transport towards the surface of evaporation, and thus controls the drying rate. The distribution of bound water content (uniform or heterogeneous) along the main sample axis and the drying rate evolution depend on the competition between the external conditions and a characteristic rate of transport due to bound water diffusion. For sufficiently slow drying this distribution remains homogenous until free water is fully extracted. An original physical phenomenon is thus at work which plays a major role of regulation of water extraction, in that it maintains a constant drying rate and a homogeneous distribution of the (mean) water content throughout the material. These results provide sound concepts for modeling and controlling drying properties of wood materials. They open the way to the understanding or control of the properties of many other materials containing two water types in food or civil engineering applications. Our results complete recent observations that bound water diffusion also controls imbibition in hardwood and finally show that transfers between bound and free water play a major role in the interaction of plant-like systems with water.
\end{abstract}

\section{Introduction}

Wood is a natural material widely used in construction, tooling, boat building, sculpture, etc. For most applications it is essential to remove a large part of its water content from wet or green (fresh-cut) wood, to reduce further dimensional variations under varying humidity conditions, improve its mechanical characteristics or protect it from biological attacks. However, drying is highly energy and/or time consuming, so that a better understanding of the internal physical processes (drying rate, dry front, type of water extracted) is essential to optimize the used techniques (e.g., kiln-drying).

Drying consists in removing water from a porous material. Here we focus on convective drying at ambient temperature, obtained by placing the wet (or green) wood sample in contact with an air flow of low relative humidity. The drying of a simple liquid layer lying on a solid surface in such a way results in evaporation from the liquid-air interface then vapor diffusion. For most porous materials with a solid structure and well- 
connected pores (or voids), water extraction may also involve vapor diffusion through the porosity but, in addition, liquid transport caused by capillary effects, as the menisci created by some liquid extraction induce a Laplace pressure that can redistribute the liquid throughout the sample. This makes it possible to distinguish characteristic drying regimes depending on the relative importance of these effects [1-7].

For wood the possibility of such basic mechanisms has long been studied, but this material has specificities which may make the process more complex [8-11]: (i) hardwoods, on which we will focus here, contain long vessels but also a large fraction of voids (fibers) whose connection with the sample free surface is tenuous or inexistent, (ii) bound water (adsorbed) in cell walls can diffuse through the solid structure. In this context, by similarity with knowledge of drying in simpler porous media, it has been considered that in a first stage some water (free water in voids) is withdrawn from the sample by capillary forces, while in a second stage drying essentially occurs by diffusion of vapor and/or bound water through the sample [9, 12-15]. However, the detailed characteristics of these two phases and the conditions of transition between them are not well identified or experimentally proved by internal observations. Then models essentially provide a phenomenological description of drying below the FSP (Fiber Saturation Point, i.e., the maximum moisture content when the wood sample only contains bound water) considering bound water transport due to a diffusion process, assuming different possible driving forces (vapor pressure, moisture content, chemical potential, etc.) at the origin of the process [16-17]. More sophisticated modeling approaches taking into account different transport mechanisms for bound water, free water and water vapor have later been developed [18-22], which appear to be able to predict macroscopic trends as soon as a number of parameters are introduced and fitted. However, they are less appropriate to provide a clear appreciation of the dominant physical mechanisms either on a global or on a local scale. This suggests that more physically sound modeling would be required, for example inspired from invasion-percolation theory [15, 23].

On the other hand, the few experimental insights in the effective internal transport phenomena during wood drying provided interesting but questioning observations. A homogeneous decrease of the water content throughout the medium was for example observed in some cases whereas a dry front developed from the free surface in other cases [23-26]. Also, it was observed that during slow drying, bound water concentration decreases when most free water has disappeared [26-28] while for fast drying bound water content decreases proportionally to free water content [29].

In order to get a clearer understanding of the physical mechanisms it appears necessary to have a view of the internal transport of water in its different states both on a global and local scale during well controlled drying of wood of known structure. Here we carried out such a study with the help of NMR (Nuclear Magnetic Resonance) measurements allowing to distinguish bound and free water contents and X-ray computed tomography (XRCT) method to image air-liquid interfaces at the smallest pore scale (wood lumens). We show that during wood drying, even well above the FSP, bound water diffusion in cell walls (and not capillary effects) ensures the extraction of liquid water from pores and its transport towards the surface of evaporation, and thus controls the drying rate. The distribution of bound water content (uniform or heterogeneous) along the main sample axis and the drying rate evolution depend on the competition between the external conditions and a characteristic rate of transport due to bound water diffusion.

These results provide sound concepts for modeling and controlling drying properties of wood materials. They show that the drying of a hygroscopic material with liquid-filled pores is controlled by the bound phase, which opens the way to the understanding or control of the properties of many other materials containing also two water types in food [30-31] or civil engineering applications [32].

Concerning plants, it is remarkable to see that bound water diffusion, which was proved to control imbibition in hardwood instead of capillarity [33-34], also controls the opposite process of drying. This finally shows that transfers between bound and free water play a major role in the interaction of plant-like systems with water.

Since the paper successively presents and interprets data obtained at different scales from observations with several techniques we start (next Section) by presenting the detailed plan of the work and results, along with the successive conclusions. 


\section{Structure of the analysis and main conclusions}

All experiments are carried out with poplar samples (hardwood). They are essentially cut along the anisotropic directions (Longitudinal L, Radial $R$ and Tangential T). The main direction of drying is along the longitudinal ( $L$ ) direction and the free surface is the transversal RT (Radial R and T tangential) plane. The structure is essentially composed of cells (vessels and fibers along the $L$ direction and rays along the $R$ direction) which contain free water and solid cell walls which may contain bound water (see Section III).

We first observe (Section IV) the (macroscopic) drying rate of wood samples subjected to an air flow along the transversal RT free surface, and see that despite the complexity of wood structure, the drying rate remains constant over almost all the process if the air flow is sufficiently low. This has two major implications: i) the conditions of liquid evaporation are maintained constant along the free surface of the sample, and ii) there exists a driving force inducing the transport of internal water towards the free surface.

MRI observations then show that the (free) water distribution remains uniform throughout the sample as long as the drying rate is constant (see Section V). This means that the driving force acts throughout the sample and impose a uniform effect over the sample length.

Usually, i.e., with simpler porous media with an impermeable solid structure, the driving force is capillarity, which leads to a constant drying rate associated with a continuous liquid network continuously reequilibrated throughout the sample and transporting the liquid towards the free surface. However, observations of the shape and distribution of the liquid-air interfaces on a local (pore) scale with 3D XRCT show that such a continuous network of free water cannot exist inside wood (see Section VI).

This implies that a different driving force has to be found. Since the main difference between wood and simple porous media is the presence of bound water inside the solid structure it is natural to consider that this bound water plays a significant role in the specific driving force involved in wood drying. In order to get an idea of this role we follow the relative amount of bound and liquid water in time by NMR relaxometry (see Section VII). It appears that the bound water starts disappearing only when all free water has been extracted and, for slow drying, the drying rate remains approximately constant even when only bound water remains in the sample.

We then discuss the physical origin of this process (Section VIII): The chemical potential gradient due to the extraction of bound water near the evaporation front (i.e., the sample free surface) is here the driving force, inducing transport of water through diffusion of bound water in the cell walls and absorption of free water in deeper cell walls. Some further evidence of these phenomena on a local scale is provided by 3D XRCT observations (Section IX): a liquid volume in a vessel progressively disappearing from this region without other possibility than absorption in cell walls.

In fact, in a structure as complex as wood the distribution of bound and free water is complex on a local scale and varies as soon as some water transport has occurred. A consequence is that the processes on a local scale are highly non-linear. Evidence is provided by observing the evolution of the distribution of filled and empty vessels and fibers in time (Section $\mathrm{X}$ ): during drying, some vessels and fibers may be emptied then refilled, while neighboring vessels or fibers follow a different evolution. A scheme of the process is proposed (Section XI).

Finally, we consider the case of a faster air flow (Section XII). In that case the velocity of water transport by diffusion in cell walls is too slow for water to replace the bound water extracted by evaporation along the free surface of the sample. From MRI and deformation measurements we show that a significant gradient of water concentration (bound and free) develops towards the interior of the sample. Water evaporation now occurs (from bound and free water) at a depth increasing in time.

The following scheme of water transport during slow drying emerges:

- Most of the evaporation occurs from bound water along the sample free surface ;

- As long as there is free water in the sample the evaporated bound water is replaced by bound water formed by absorption of deeper free water in the cell walls and diffusion towards the free surface;

- When there is no more free water the drying goes on by progressively decreasing the bound water content in the sample, thus progressively forming a dry front. 
For fast drying the diffusion of bound water through cell walls is not sufficiently rapid, which leads to the development of a gradient in the bound water distribution earlier in the process.

\section{Methods}

\section{III.1 Materials}

Green wood samples for NMR experiments $\left(R \times T \times L=6 \times 6 \times 10 \mathrm{~mm}^{3}\right)$ and for X-ray computed tomography experiments $\left(R \times T \times L=4.5 \times 4.5 \times 40 \mathrm{~mm}^{3}\right)$ are collected from cut-off poplar trees in the Region Nouvelle Aquitaine (Biron city). The sample for MRI experiments $\left(R \times T \times L=38 \times 16 \times 38 \mathrm{~mm}^{3}\right)$ is collected from poplar plank, which had been left drying naturally under ambient conditions (from Region Ile de France). All samples are sawn along the anisotropic directions of wood (longitudinal L, Radial R and Tangential T) and come from the inner part of the trunk (heartwood).

Poplar (and more generally hardwood) is basically made of long (hollow) dead-end vessels extending in the L-direction (see Figure 1) and whose diameter is about $100 \mu \mathrm{m}$, through a solid structure containing elongated enclosed fibers (also in the L-direction) which can store some free water. Their length ranges from 1 to $12 \mathrm{~mm}$ and their diameter is about $10 \mu \mathrm{m}$. Neighboring vessels may be connected by pits, which are membranes with pore size of a few tens of nanometers. Pits between fibers are generally inconspicuous. There are also some rays, made of a series of parenchyma cells (approximately $15 \mu \mathrm{m}$ high, $10 \mu \mathrm{m}$ wide and $200 \mu \mathrm{m}$ long), aligned along the radial direction ( $R$ ), which may be connected to fibers and vessels, thus creating a privileged pathway for water and biochemicals in the radial direction. The volume fractions of vessel pores, fibers pores and ray pores, accessible to free water, are approximately $34 \%, 55 \%$, and $11 \%$. This leads to a porosity of about $68 \%$ (for the maximum sample volume, i.e., saturated with bound water). Moreover, the cell walls can absorb water in the form of bound water, up to a moisture content of about $30 \%$.

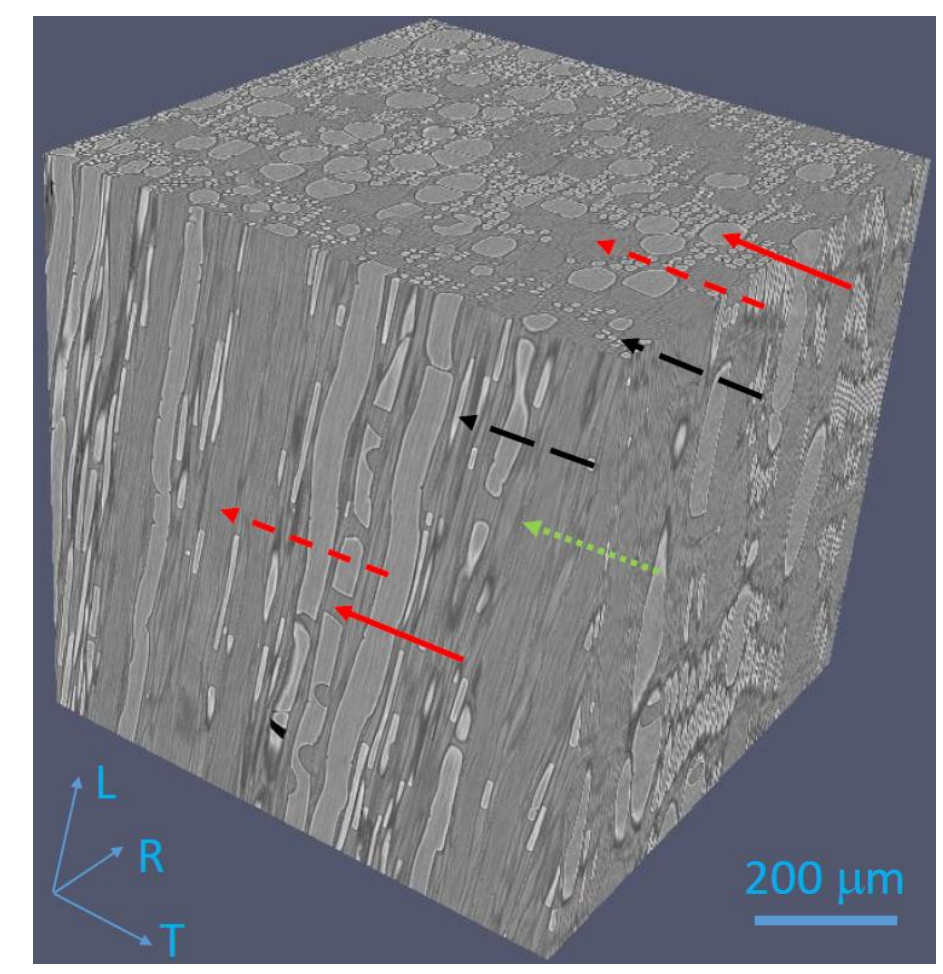

Figure 1: 3D XRCT view of the wood structure during drying (at $M C=151 \%$, see drying characteristics in Section XII). Light gray correspond to air regions, darker gray areas correspond to liquid water or cell walls. Red arrows show the vessels, black (long dashed) arrows the fibers, and green (dotted) arrow the rays. Short dashed red arrows show vessels containing water. 
The wood samples are taken in a region as homogeneous as possible, and are tested either green, or resoaked after drying. Although the resulting values of initial water content in vessels and fibers differed from one sample to another the trends observed by the different techniques and the mechanisms discussed below were qualitatively similar. Under these conditions we can consider that we are dealing with a single given material and focus on its physical properties.

\section{III.2 NMR relaxation and 1D profiles}

The sample is inserted in a Bruker NMR minispec mq20, $0.5 \mathrm{~T}$ (for tests a), b) and c)). We measure the NMR relaxation through a Carr-Purcell-Meiboom-Gill (CPMG) sequence composed of a first $\pi / 2$-pulse and $1000 \pi$ pulses during $5000 \mathrm{~ms}$ distributed in logarithmic intervals typically between 0.5 and a few tens of ms. The repetition time is $10 \mathrm{~s}$ to get a complete relaxation of all protons. This sequence is repeated 16 times to increase the signal to noise ratio. The $T_{2}$ distribution can then be resolved by means of ILT (Inverse Laplace Transform). Our procedure is a non-negative least square fit to the data with Tikhonov regularization, which is similar to the 'Contin' method [35-36], and described in [37]. We finally get an apparent statistical distribution of $T_{2}$, expressed in terms of signal intensity associated with each possible value of $T_{2}$, which we will call here "T distribution". Note that the exact shape of this T distribution depends on the technique of regularization used, and in particular on the value of the "parsimony factor" [37]. However, the positions of the peaks and the relative amounts of liquid associated with these peaks are robust values. Also, the evolution of the shape of the distribution is reliable information that can be used for a qualitative physical analysis, as long as the parsimony factor is kept constant for the treatment of all corresponding data. As we are looking at the signal resulting from protons of mobile hydrogen atoms we consider that there are no more water molecules (either free or in bound state) inside the sample wood when the NMR signal is equal to zero.

In addition, roughly speaking the relaxation time is related to the mobility of water molecules, and specific interactions of water with their environment (e.g. adsorption, proton lability, presence of paramagnetic elements, etc). In the particular case of free water embedded in a pore cavity, within the usual hypothesis of biphasic fast exchange [38], the relaxation time scales as the ratio of the volume of free liquid water to the area of the water-solid interface, with a factor depending on the NMR surface relaxivity [39]. Note that this volume-to-surface ratio is only proportional to radius - not only for uniform spherical pores but also for cylindrical pores (i.e., no dependence on the cylinder length). We deduce that a more or less filled cylinder yields a fixed relaxation time, while a cylinder with a progressively thinning liquid layer (along its walls) yields a decreasing relaxation time. Also, a homogeneous decrease of liquid content in a pore class leads to a decrease of the peak amplitude, and the curve portion is directly shifted towards lower relaxation times. In contrast, a heterogeneous spatial variation of the liquid content leads to a spreading of the peak towards lower values [40]. For bound water, the relaxation time depends on the average mobility of the bound water. Different types of bound water may be distinguished but here we simply assume that the local relaxation time decreases with the total concentration of bound water. Each of the " $\mathrm{T}_{2}$ distributions" may be analyzed by assuming that the liquid in the vessels, if filled with liquid, has a relaxation time around $600 \mathrm{~ms}$, the liquid in the fibers has a relaxation time around $80 \mathrm{~ms}$, and the relaxation of bound water is typically in the range 0.5 to $5 \mathrm{~ms}$. Note that a straightforward correspondence between these values and the pore size cannot be established without knowing the relaxivity of the medium, but the order of magnitude of these values is consistent with various observations. Then the amount of liquid of each class may be estimated from the integral of the $T_{2}$ distribution around the peak of relaxation time associated to this class. This approach is precise as long as the overlap between the peaks is sufficiently small.

For the 1-cm-long samples it was also possible to get 1D NMR profiles with the same NMR system equipped with a $4 \mathrm{~T} / \mathrm{m}$ vertical pulsed gradient unit. The profiles were performed with 128 pixels over a field of view of $2 \mathrm{~cm}$ performing a multi-echo sequence ( $\mathrm{TE}=7.2 \mathrm{~ms}$ ) with 128 echos set during 1 second, and 64 accumulations (to increase the signal to noise ratio) and a repetition time of $8 \mathrm{~s}$. A Fourier transform of the data for each echo time is then processed. For each pixel, the water signal is finally given from the amplitude 
of an exponential fit to the data associated with the 64 1D profiles corresponding to each even echo (to remove $\pi$ pulse imperfections). Furthermore, a calibration of each profile is set by a pure water filled tube to remove effects of magnetic heterogeneities. Note that, with this technique, the bound water with its very short relaxation should essentially be visible in the first (odd) and the second (even) echo, which negligibly impact the amplitude deduced from the exponential fit. As a consequence, we can consider that the NMR signal corresponding to bound water is of the same order or smaller than the noise during all the drying of free water. Bound water is thus not detected by this sequence. This is confirmed by the excellent consistency of the total NMR signal deduced from 1D profiles and the free water amount deduced from relaxometry (see data below). At last, the technique and setup described by Zhou et al. [33] was used to get 1D MRI profiles on large samples. In that case, the large length of the sample allowed making direct measurements of the deformation, which can be used to deduce the amount of bound water in each section [33].

\section{III.3 X-ray micro-computed tomography imaging}

Experiments were run on the Anatomix beamline of Synchrotron SOLEIL during the beamtime of proposal No. 20180522. Anatomix is a 200-m-long beamline using a cryogenic in-vacuum undulator source (U18) which allows using various tomographic setups for 3D imaging over a wide range of length scales and materials, and provides absorption and phase contrast [41]. In the present study, a rather classical parallelbeam absorption tomography setup was used and local tomography was performed. The undulator was used with a gap of $13 \mathrm{~mm}$ and the beam was filtered by a 0.9-mm CVD diamond filter resulting in a largeband "pink" beam centered at a photon energy around $15 \mathrm{keV}$. The $\mathrm{X}$ rays were converted into visible light with a lutetium aluminum garnet (LUAG) scintillator mounted in front of a $45^{\circ}$ mirror, which redirects the light into a $5 x$ Mitutoyo objective that creates a magnified image of the scintillator plane on the CMOS sensor of an ORCA Flash $4.0 \mathrm{~V} 2$ camera from Hamamatsu. The voxel size of the reconstructed volumes is $1.3 \mu \mathrm{m}$ with a field of view of $2.6 \mathrm{~mm}$; volume dimensions were $(2048 \times 2048 \times 2048)$ voxels. The spatial resolution of this setup and the contrast obtained were sufficient to image liquid inside lumens (voids) and to cover a large part of the growing ring. A typical acquisition sequence includes the recording of dark images, flat-field images for normalization before and after the scan, and a $180^{\circ}$ scan of the sample containing 2,000 projections (i.e., angle between projections was $0.09^{\circ}$ ).

A special device has been developed to perform "in-situ" experiments, taking into account the constraints of the Anatomix beamline and the variation of sample dimensions with moisture content: a container cast by $3 \mathrm{D}$ printing with flexible clips to vertically maintain the samples and to take into account the variation of sample dimensions during the drying process. Before a drying test, the lateral surface of the sample was covered with parafilm ${ }^{\circledast}$ tape and the reference image is the first acquired image. The evolution of liquid distribution in the sample was obtained from a succession of images during the experiments over a period of $70 \mathrm{~h}$. Local tomography was performed at two heights (at 5 and $10 \mathrm{~mm}$ from the top free surface). The duration of each tomography scan was 200 s. Successive acquisitions (i.e., over a very short period) at some time during the process have also been performed, allowing a further insight in the liquid transfer dynamics and the drying mechanisms. The samples were weighed before and after each test to check that the X-ray synchrotron radiation does not modify the drying dynamics (on a "macroscopic" scale) within $0.1 \%$. All volumes were reconstructed with the software PyHST2 [42] using a Paganin filter, and first analysed with the Fiji software [43]. To compare images at successive times, similar positions were identified by inspection of the structure (vessels and fibers). Then the gray level range was improved and a filter applied with ImageJ software. In some cases a series of images were colored differently depending on the observed evolutions and then superimposed using Draw (LibreOffice) software. 


\section{III.4 Drying tests}

The samples were either in fresh-cut state or dried then re-soaked by immersion in water for a few days, then covered with an impermeable tape on all sides except the top surface. The temperature is maintained at $22{ }^{\circ} \mathrm{C}$. The samples are either subjected to a (vertical) dry air flux towards their free surface, or left in ambient air at a $\mathrm{RH}=50 \%$. The flow rate of the air flux (typically of the order of $10^{-3} \mathrm{~m}^{3} \mathrm{~min}^{-1}$ ) for the NMR experiments, and its position in the NMR tube could be varied, leading to different rates of evaporation. These drying conditions are anyway only indicative as the resulting drying rate also significantly depends on the exact flux orientation and shape and roughness of the sample surface. Under these conditions the relevant parameter describing the external conditions, which are in any case convective, is the initial drying rate. Indeed, since the sample is initially wet along its free surface, the initial drying rate directly describes the evaporation rate of the water molecules covering the top surface, which is a function of all boundary conditions mentioned above (temperature, flow rate, air flux orientation, $\mathrm{RH}$, roughness, etc).

We take wood samples in which all faces but one are covered with an impermeable tape so that water can be extracted in the form of vapor only through the RT (Radial-Tangential) free surface (see Figure 2). We thus here look at the drying in the longitudinal direction, i.e., along the main axis of the vessels and fibers.

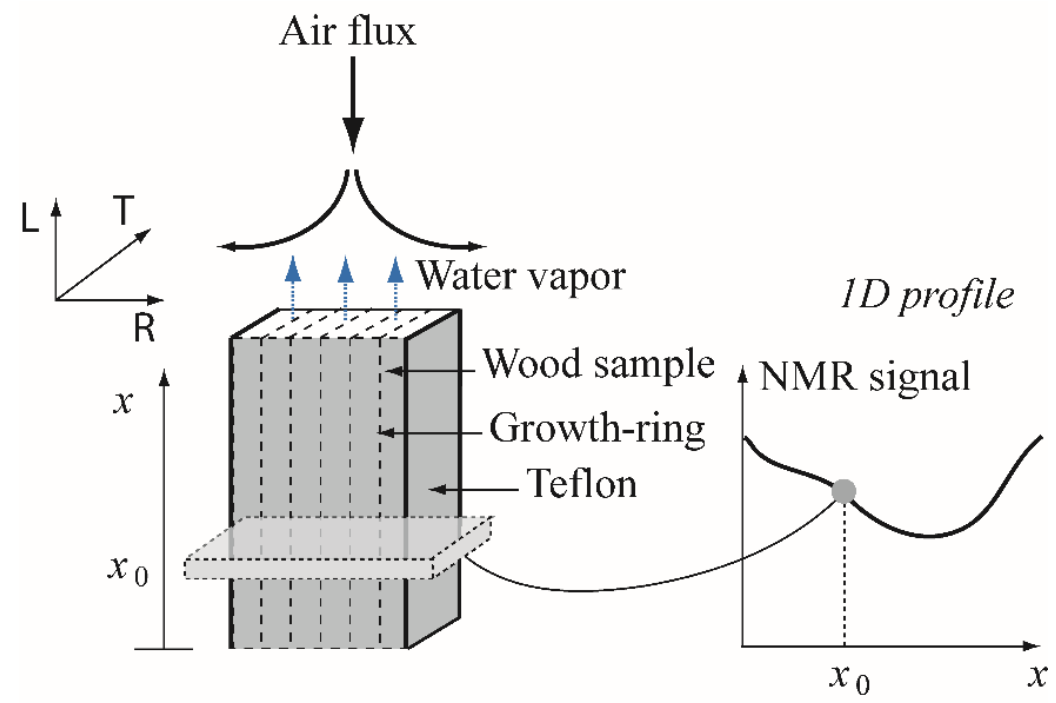

Figure 2: Scheme of the main aspects of the setup for drying experiments: (left) sample orientation for drying and main axes, where $\mathrm{R}$ and $\mathrm{T}$ are the radial and tangential directions and $L$ the longitudinal direction (vertical tree axis); (right) principle of the 1D NMR profile measurements, where the data point for $x_{0}$ in the profile corresponds to the total NMR signal in a thin layer of the sample situated at $x_{0}$.

The water content of the whole sample in time is followed by NMR (see above), which allows to measure the total mass of water whatever its state (bound or free). We then can compute the drying velocity (or drying rate) $(V)$ defined as the liquid volume crossing the sample free surface per unit time $(t)$ and surface $(S)$. In fact, the water leaves the sample only in the form of vapor. Thus, the drying rate results from the evaporation along some liquid-air interfaces inside or at the surface of the sample, and this vapor is then transported by diffusion through the sample free surface. In this context, liquid transport inside the sample, which induces a redistribution of the water-air interfaces, can play a significant role in the process. As a consequence, the drying velocity is a marker of the different mechanisms and may be directly analyzed to identify the dominant mechanism. Usually, for simple porous samples of cylindrical or cuboid volume $\Omega$ and height $H$ with a pore volume $\varepsilon \Omega$, where $\varepsilon$ is the porosity, we have $V=-\varepsilon H \mathrm{~d} \psi / \mathrm{d} t$, where $\psi$ is the saturation, defined as the volume fraction of liquid in the pore volume. For wood samples, as the pore volume is difficult to estimate, the samples may be initially partially saturated and water may be in different states, we describe the water 
volume through the moisture content, $M C$, i.e., the ratio of water mass to the mass $(m)$ of the dry sample, so that the drying velocity (or drying rate), as defined above, here writes:

$V=-\frac{m}{\rho S} \frac{\mathrm{d} M C}{\mathrm{~d} t}$

where $\rho$ is the liquid water density (pure water under ambient conditions). Note that the drying rate is computed from the mass variation over time. As a consequence, it exhibits noise which is a function of the uncertainty on mass measurement and of the time interval chosen. The scatter observed on the drying rate values (see, e.g., Fig.3) reflects the uncertainty on data.

\section{Drying rate}

The first fundamental observation is that during a first period of drying the drying rate is constant, and starts to decrease below some critical moisture content, whose value decreases for decreasing initial rate of evaporation (imposed by boundary conditions) (see Figure 3). In other words, all other things being equal the initial constant drying rate period is longer for slower drying.

Although such a macroscopic observation (constant drying rate) may appear trivial, it is in fact surprising because it means that despite the transient nature of the process, i.e., the water content is progressively decreased inside the sample, which implies that the potential surfaces of evaporation change, a constant global evaporation rate is observed. As a consequence, this result (constant drying rate) is significant from a physical point of view: as explained below it tells us a lot about the behavior of the liquid in the structure over time.

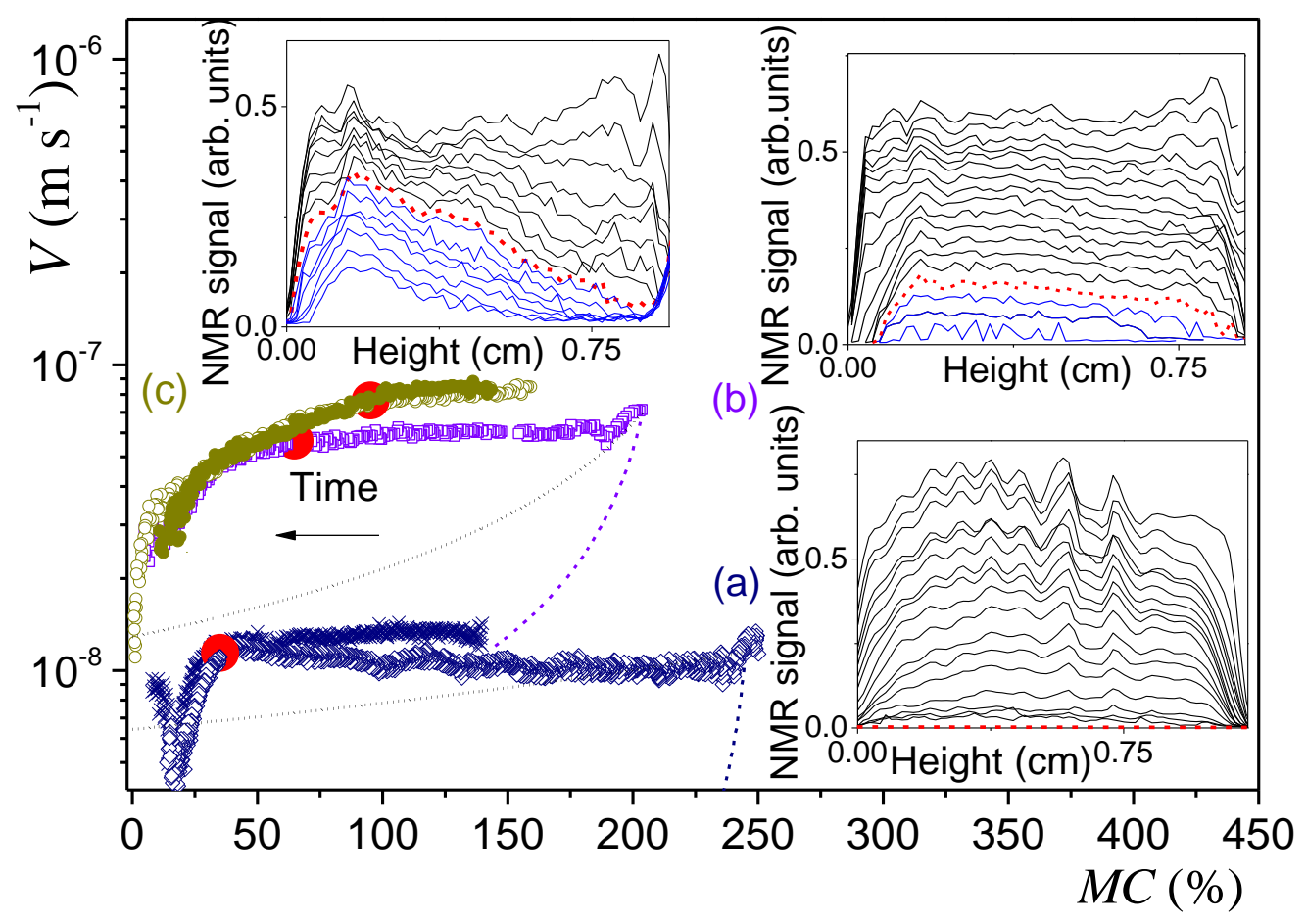

Figure 3: Drying rate of wood samples ( $1 \mathrm{~cm}$ along L-axis) as a function of the moisture content for different initial drying rates (corresponding to the first values on the right of each curve) (for all these tests we had $\Delta n=1$, i.e., a flow of completely dry air): test (a) (dark blue diamonds); test (b) (violet squares); test (c) (dark yellow circles). The filled symbols show a similar test with the same sample (open symbols of same color) dried after re-soaking for case (c). The dark blue crosses show a test similar to case (a) but with another sample at a different initial MC (see 
Appendix B). The insets show the 1D NMR profiles of free water measured at different times during each of these three tests: (from top to bottom) (a) every $11 \mathrm{~h}$, except the three last profiles every $3 \mathrm{~h}$, (b) every hour, (c) every 30 min starting from $2 \mathrm{~h}$ after the beginning. Let us recall that each point in these profiles corresponds to the total excitation of hydrogen proton spins contained in a small layer of sample situated around this position (see Figure 2). The red dashed line in NMR profiles (insets for cases $a, b$ and $c$ ) is the last profile for which free water exists throughout the whole sample (black profiles before, blue profiles after this profile). The corresponding points on the drying curves are indicated as red disks. The dashed lines correspond to the model of drying front in vessels only (equation (4)), the dotted lines to the dry front model for all water (equation (5)).

A constant drying rate means that the conditions of evaporation do not evolve significantly during the process. In order to analyze the implications and thus give a more precise description of the physical meaning of these "conditions of evaporation", let us review some basic principles of (convective) drying at ambient temperature. For a porous sample almost filled with liquid water we can consider that, at the beginning of drying, a lot of liquid-air interfaces, resulting from liquid "patches", are situated along the free surface of the sample, so that the relative humidity (RH), here denoted $n$, along the free surface is close to 1 as a first approximation. Then drying results from the vapor diffusion from this free surface to a region where $n=1-\Delta n<1$. The process may be generally described by considering that this diffusion occurs through a boundary layer (of air) of thickness $\delta$ [44], whose value is smaller for stronger air flux. Under such conditions, from Fick's second law, the drying rate can be written as

$V_{0}=\frac{\rho_{0}}{\rho} \frac{D_{0} \Delta n}{\delta}$

with $D_{0}=2.7 \times 10^{-5} \mathrm{~m}^{2} \mathrm{~s}^{-1}$ the water vapor diffusion coefficient in air, and $\rho_{0}=0.0234 \mathrm{~kg} \mathrm{~m}^{-3}$ the maximum vapor density in air (at $25^{\circ} \mathrm{C}$, for $n=1$ ). For example, for the test (b) of Figure 3, from the measured drying rate at the initial times and the above expression we deduce $\delta \approx 1 \mathrm{~cm}$. Thus, this single parameter $\delta$ directly describes the impact of the boundary conditions, including $\mathrm{RH}$ and air flux, on the drying rate, whatever the convective drying technique used.

From this description we see that a change of position of the liquid-air interfaces may lead to a modification of the value of $\delta$, which will induce a change of the drying rate. Let us for example assume that for a porous medium some dry region of thickness $h$ develops inside the sample from its free surface, so that the liquid now evaporates exactly from this depth (corresponding to the new position of the liquid-air interfaces) and the vapor then diffuses to the free surface over the distance $h$, before reaching the external boundary layer of thickness $\delta$. Now the relative humidity at the sample free surface is $n_{1}$ and (assuming for the sake of simplicity $\Delta n=1)$ the drying velocity is $V=n_{1} \rho_{0} D_{0} / \rho \delta=n_{1} V_{0}$. Note that from this approach, $V_{0}$ is the initial drying rate. Besides, the vapor density gradient along the dry region induces a vapor flux which can be expressed in steady state as $V=\rho_{0} D_{v}\left(1-n_{1}\right) / \rho h$, where $D_{v}$ is the vapor diffusion coefficient through the (dry) porous medium. We can write $D_{v}$ as $\varepsilon D_{0} / \tau$, where $\varepsilon$ and $\tau$ are the porosity and the tortuosity of the medium [45]. From the two expressions above for the vapor flux we deduce $n_{1}=1 /\left(1+D_{0} h / D_{v} \delta\right)$, and the drying rate can be written

$V=\frac{\rho_{0}}{\rho} \frac{D_{v}}{\left(h+\delta^{*}\right)}=\frac{V_{0}}{\left(1+h / \delta^{*}\right)}$

with $\delta^{*}=D_{v} \delta / D_{0}$. This model has been validated, for example, in the context of the drying of model porous systems (uniform bead packings with bead size from 45 to $0.1 \mu \mathrm{m}$ ) for which both the drying rate and the thickness of the dry region could be measured in time [46].

Considering hardwood structure, the simplest assumption is that the liquid contained in the vessels (large paths connected to the free surface) dries first, so that the liquid-air interface withdraws towards the interior and the vapor then essentially diffuses through the (straight) vessels (thus $\tau=1$ ) along a distance $h$. In this 
context, we can for example consider two different situations: either a first full drying of the vessels without any transport of the rest of water (i.e., contained in cell walls or fibers), or by assuming that at each step all the bound and free water situated down to the depth $h$ has been extracted and transported towards the free surface by (vapor) diffusion along the vessels from this depth, thus forming a fully dry front.

Let us first assume that the vessels are initially filled with water and that they dry first, while the remaining water (contained in fibers and cell walls) is not transported at all. In that case the water evaporates from the liquid-air interface and diffuses along the vessel towards the free surface of the sample, and the liquid-air interface progressively advances deeper in the vessel. The drying rate is given by (3), in which we have to relate $h$ to the moisture content. The initial moisture content associated with vessels is $M C_{v}=0.34 \times\left(M C_{0}-M C_{b}\right)$, where $M C_{0}$ is the moisture content associated with saturation and $M C_{b}$ the maximum moisture content associated with bound water. We deduce in this case $h=H\left(\left(M C_{0}-M C\right) / M C_{v}\right)$ and the final expression for the drying rate is

$$
V=\frac{V_{0}}{1+\frac{H\left(M C_{0}-M C\right) \rho V_{0}}{\rho_{0} D_{v} M C_{v}}}
$$

Note that here $D_{v}=\varepsilon D_{0}$ with $\varepsilon=0.34 \times 0.68=23 \%$ being the porosity associated with vessels, since $\tau=1$ for vapor diffusion in straight channels.

Alternatively we can assume that, when the liquid-air interfaces are situated at a depth $h$ in the vessels, all the water, i.e. bound and free water, situated above this level in the sample, has been extracted. More precisely, we assume that at each step the water situated around the depth $h$ is extracted and transported towards the sample free surface by vapor diffusion along the vessel. In this scheme a straight dry front grows from the free surface towards the interior of the whole sample, and we have $h=H\left(\left(M C_{0}-M C\right) / M C_{0}\right)$, so that

$$
V=\frac{V_{0}}{1+\frac{H\left(M C_{0}-M C\right) \rho V_{0}}{\rho_{0} D_{v} M C_{0}}}
$$

In both cases, i.e. dry front in the vessels or in the whole sample, the drying rate is not constant, which is in contradiction with our observations. Finally, the predictions of these models (equations (4) and (5)) significantly depart from the observed drying rate (see Figure 3). Thus, any type of dry front growing from the sample free surface towards the interior of the sample, leads to a non-constant drying rate which differs from our observations.

Actually, the constant drying rate observed in these tests is the hallmark of evaporation conditions remaining constant during all this period, i.e., the spatial distribution of relative humidity, imposed by the air flux and the distribution of liquid-air interfaces, remains constant. This has two important implications on the drying characteristics of this constant rate period:

(i) Sufficiently wet conditions around the free surface of the sample are preserved, i.e., similar to those prevailing at the beginning of drying;

(ii) Sufficient water is continuously transported towards the free surface to maintain these wet conditions.

In the next sections ( $\mathrm{V}$ to $\mathrm{XI}$ ), the mechanisms of drying in the constant drying rate regime are analyzed. The transition in drying rate regime occurring at sufficiently low $M C$ will be discussed in Section XII.

\section{Water content distribution during drying at constant rate}

In order to transport the water towards the free surface of the sample it is necessary to have a sufficiently large driving force acting over almost the entire process to replace the liquid extracted as a result of evaporation at the free surface. The existence of such a large driving force implies that the emptying of the 
sample should occur homogeneously, as this force should also act in any region to balance out some liquid depletion. This is effectively what we observe from 1D NMR profiles (see insets in Figure 3): the (free) water content along the height in the sample decreases homogeneously in time (i.e., its distribution remains approximately uniform) during the constant rate regime. Thus, some equilibrium is maintained throughout the sample as long as free water is extracted.

Such characteristics are observed with simple porous systems $[3,46]$ containing only free water, during the so-called "Constant Rate Period": a continuous liquid network is preserved, which enables liquid transport towards the free surface through capillary effects tending to balance out the saturation throughout the medium. This finally maintains a sufficient density of liquid patches close to the free surface, which induces a RH close to 1 along the free surface, even if the typical patch size decreases as drying progresses [47]. The liquid transport towards the free surface requires good wetting of the solid surfaces by the liquid (say, a contact angle inferior to $90^{\circ}$ ) and, at any time, a continuous liquid film covering the solid surface and connecting the whole liquid phase. Note that a continuous liquid network is essential in this context to ensure the transport [48-49]: if the liquid volumes are disconnected, the only possible transport of water is in the vapor state, but such transport is negligible because the relative humidity inside a region surrounded by liquid patches is constant and equal to 1, thus precluding diffusion (see, e.g., [48-49]).

It is thus interesting to check whether such a liquid network of free water exists during wood drying. It can rather easily exist in simple porous media such as bead packings since the pore network in these materials is made of pores of similar size well connected to the others through paths of size of the same order. The pore network of wood is very different, as it is made of open (vessels) and dead-end (fibers) conduits, only possibly connected via some very small pits with nanometric holes. In this context it is even more remarkable that for a sufficiently slow drying (case (a)) the 1D NMR profiles remain approximately uniform up to the complete disappearance of free water (see inset (a) of Figure 3). Such an effect does not even occur in simple porous media such as bead packings for which a dry front starts to develop at the latest when there remains $10 \%$ of the initial water (initially saturated sample) [46].

\section{Shape and distribution of liquid-air interfaces}

In order to search for the possible existence of a liquid network we look at the water distribution at the micron scale, from XRCT observations of air-liquid interfaces of the poplar sample (see Section III). From an inspection of the transitions of gray level, resulting from different X-ray attenuations, we can identify the position and shape of the air-liquid interfaces (see typical example in Figure 4). Some of the vessels are filled with water, at least in the observed region, while some others are apparently partially empty. Some fibers are partially filled, with liquid filling one of their extremities (blue arrows in Figure 4). 


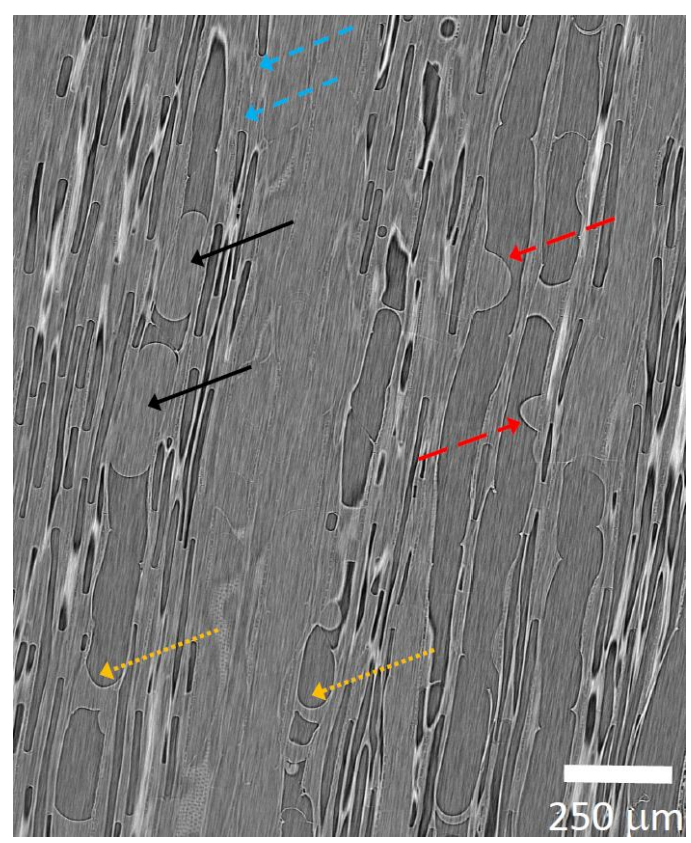

Figure 4: Typical view of the internal structure of a wood sample (sample length: $4 \mathrm{~cm}$ ) at 3.7 $\mathrm{mm}$ (mean distance) in a TL plane (see Fig.2) below the free surface during drying in the constant rate regime $(M C=151 \%)$ (see drying characteristics in Section XII). The darkest areas correspond to air. The lightest gray areas correspond to free water or cell walls containing bound water. The (short dashed) blue arrows show water in partially filled fibers. The black arrows show liquid drops blocking a vessel and with a contact angle around $150^{\circ}$. Red (long dashed) arrows show liquid drops on one side of a vessel and with a contact angle around $80^{\circ}$. Yellow (dotted) arrows show liquid drops blocking a vessel and with a contact angle around $0^{\circ}$. Note that in a 2D picture vessels can easily be distinguished from fibers as their diameters are typically ten times larger than fiber diameters. A 2D plane can occasionally cross a vessel along its side, leading to an apparent channel of size similar to that of fibers, but this is a rare event.

The angles between the liquid-air interfaces and the vessel or fiber walls in 2D XRCT images were measured by fitting a disk of appropriate diameter to the interface in the neighborhood of the point of contact, and estimating the slope of the tangent at this point (see Appendix A). The uncertainty on this estimation is about 10 degrees. These measurements were carried out on 160 interfaces in vessels and 150 interfaces in fibers taken in various places in the imaged volume, in images of the same type as Figure 3 (see two other examples in Appendix A). We only considered interfaces having a sufficiently clear intersection with the wall. Note that the sharp apparent interfaces in some fibers in fact correspond to the end of the fiber (i.e. solid-air interface). The distribution of angles is represented in Figure 5. We thus obtain apparent contact angles, which just give some indication on the effective contact angle in the absence of more information on the rest of the interface shape. However, when the interface is circular in the plane of observation and in contact with both sides of the vessel or fiber, due to the relation between the curvatures and the pressure drop (Laplace pressure), and the uniformity of the pressure in the liquid drop in the absence of gravity or inertia effects, it follows that the curvature in a perpendicular plane is constant; we deduce that the interface shape is either cylindrical with an axis perpendicular to the plane of observation, which is incompatible with the shape of vessels and fibers, or spherical. Here we observe that most of the interfaces (say, about $80 \%$ ) in fibers are circular, while only about $60 \%$ of the interfaces in vessels are circular, which means that large fractions of these interfaces are spherical caps. 


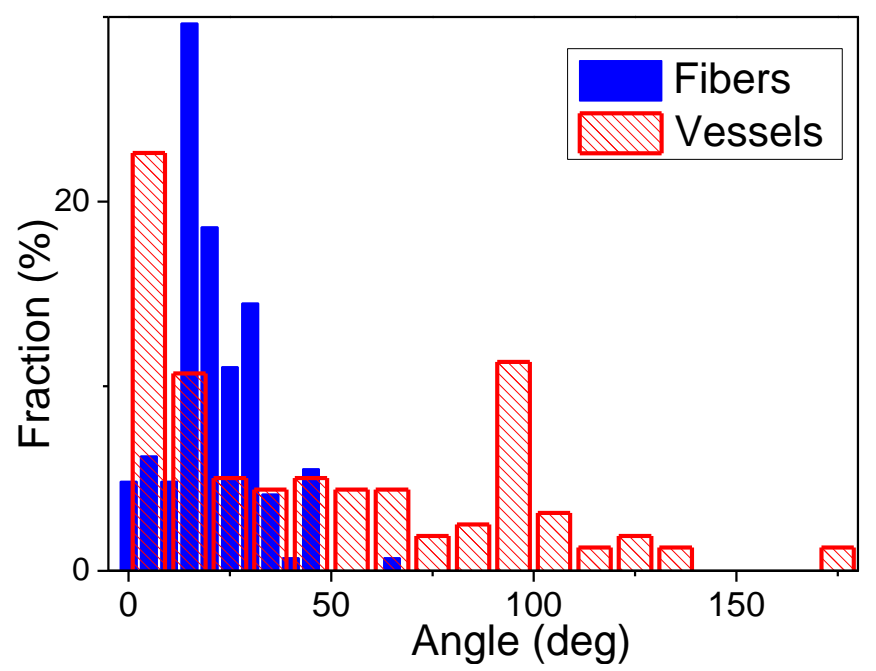

Figure 5: Fraction of liquid-air interfaces in vessels and fibers as a function of the angle range $\left(10^{\circ}\right.$ steps for vessels, $5^{\circ}$ steps for fibers) from the successive images obtained at $M C=151 \%$.

In fibers, which can be approximately considered as cylinders, the liquid-air interfaces are essentially spherical caps (see Figure 4), and the contact angle distribution is gathered around a peak at about $20^{\circ} \pm 10^{\circ}$ (see Figure 5). Note that this is nevertheless an apparent contact angle, as there might be some pinning effect, as a result of the wall roughness or the perforations forming rims, which can induce a significant straining of the interface.

In partially filled vessels, the liquid-air interfaces take the form of drops of liquid with various shapes, with an apparent angle of contact in a much wider range than in fibers (see yellow to red then black arrows in Figure 4). Actually two peaks appear in the distribution of contact angles: around 0 and around $90^{\circ}$ (see Figure 5). Note again that the same pinning effects as mentioned above can occur, along with the strong effect on wetting properties recently observed [33-34]: it was indeed shown that the contact angle of the liquid-air interfaces in the vessels is around $90^{\circ}$ when water comes into contact with non-saturated cell walls, so that imbibition is strongly slowed down, while it is much smaller when the cell walls are saturated, allowing fast imbibition. In any event, here we will not analyze the origin of the values of these apparent contact angles; we will rather discuss the implications concerning the liquid distribution in the porous structure, and the possible water transport by liquid flow.

At first sight, no clear hydraulic connection seems to exist between the liquid patches. In particular, we do not directly see liquid films along the walls in the rest of the partially filled fibers and vessels, but considering the resolution of our images (voxel size of $1.3 \mu \mathrm{m}$ ), a film of thickness of a few microns or smaller could exist. Actually, it is worth having in mind that in a cylindrical vessel, a drop forming a meniscus with contact angle $\theta$ can be connected with a film along the walls and at (capillary) equilibrium only if the Laplace pressure is the same along the liquid film and in the drop. This implies that we need to have an equality between $\sigma / R$ (Laplace pressure in the film) and $2 \sigma \cos \theta / R$ (Laplace pressure in the drop), which implies a specific value for $\theta$, namely $60^{\circ}$. As a consequence, for fibers and vessels, which are approximately cylindrical and for which the contact angle is either clearly different from $60^{\circ}$ (for fibers) or takes various values with two peaks at 0 and $90^{\circ}$ (for vessels), we should in general not have the possibility of films.

Note that the demonstration above is valid in the absence of pinning, but if the line of contact is pinned, by definition it is not connected with a film. This nevertheless does not totally exclude the possibility of complex shapes with lines of contact pinned along almost longitudinal contact lines allowing the existence of some film covering a fraction of the wall surface. Such a situation is typically observed for a rectangular container, with liquid films remaining pinned to the exit corners [48], and maintaining a liquid transport towards the 
exit and a constant drying rate. Such a situation seems rather unlikely with our system. Indeed, the vessel cross sections are generally elliptical, with some possible unevennesses. Such unevennesses (in particular certain contiguous vessels) can occasionally permit the existence of a local longitudinal film (covering the unevenness, with the same total curvature as the main liquid-air interface, for capillary equilibrium), but since in these natural materials the vessel shape at least slightly changes all along its axis, such a film could hardly exist along all the vessel, up to the free surface. Another element of evidence arises from the absence of any film or junction between the main liquid volume and a film, in any of the vessels in our pictures.

A hydraulic connection between the liquid patches in the vessels and the sample top (free surface) is thus very unlikely. This is, a fortiori, the same for the liquid in fibers which could be connected to the free surface of the sample only through possible small pits with nanometric holes between them and the vessels, and then through liquid films of free water in partially filled vessels. In any event, such connections between liquid in partially filled fibers and vessels can hardly exist in quasi-static conditions (which prevail in such slow drying), as they would not be compatible with capillary equilibrium since there is in general a large ratio of meniscus radii between the vessels and the fibers. We can nevertheless remark that there might subsist very thin films along the cell walls, typically of the order of a few molecules thick, which would not be stabilized by standard capillary effects but by disjoining pressure and local RH. However, their possible transport would be extremely slow, and these molecules might hardly be distinguishable from the bound water, so that they would behave more or less like bound water in terms of transport. We conclude that in general the liquid volumes of free water in the porous structure of the wood do not form a continuous hydraulic network allowing, as in bead packing, the transport of free water towards the free surface. This implies that we have to look for another mechanism to explain the transport of water from the interior of the sample towards the free surface.

\section{Bound and free water dynamics followed by NMR relaxometry}

Since the specificity of wood as a porous medium is the presence of bound water in the solid structure, it is natural to consider that bound water may play a significant role in the driving force at the origin of free water transport. In order to get an idea of this role we look at the evolution of the different types of water in the sample during drying. This is possible thanks to the NMR relaxation time which decreases when the pore size decreases or, more generally, when the water mobility is reduced. We measured the distribution of relaxation times at different times during drying (inset of Figure 6, see also Appendix B). In the initial distribution we can identify mainly three peaks, respectively associated with free water filling vessels $\left(T_{2}\right.$ around $700 \mathrm{~ms}$ ) or fibers ( $T_{2}$ around $\left.100 \mathrm{~ms}\right)$, and bound water ( $T_{2}$ around $\left.4 \mathrm{~ms}\right)$. Also note a peak of small amplitude ( $T_{2}$ around $20 \mathrm{~ms}$ ) which might correspond to water in wood rays, but for the sake of simplicity and because the two peaks (fibers and rays) are close we will include them in the "fiber group".

From these data and in the case of a long constant-rate period (i.e., case (a) in Figure 3), we clearly observe that, during a first stage (A), the vessels and the fibers drain simultaneously: the amplitude of the peaks corresponding to water in these regions progressively decrease (see inset of Figure 6). At the end of this stage the vessels are empty while a significant volume of liquid remains in the fibers. In the next stage (B) the fibers drain until they are fully empty. This can be analyzed more quantitatively by integrating the signal below each peak associated with the different water classes, which allows following the evolution of the fraction of water in each class (see Section III). The results are shown in Figure 6 for the case (a) of Figure 3. In addition we show in Appendix B the results for the similar test with another sample at a different initial MC. Very similar trends are observed, which supports the generality of the conclusions presented below under slow drying conditions.

We can see that during the two stages described above the bound water amount remains approximately constant (see Figure 6; a slight increase is nevertheless observed for which we have no straightforward explanation as yet). Finally, the essential decrease of bound water amount starts in the third stage (C), when 
all free water has disappeared. It is worth noticing that, as long as free water is removed (stages A and B), the relaxation times of water in vessels or in fibers remain constant, i.e., the positions of the peak remain approximately fixed (see inset of Figure 6), while the water content in both regions decreases, i.e., the integral of the curve below the peak decreases. This means that the liquid keeps forming drops of similar sizes, or more precisely similar aspect ratios, while drying progresses. In contrast, if water was forming films or blobs of thickness of progressively decreasing thickness in time, the relaxation time would decrease significantly (see Section III). This is consistent with the observations of Section VI, and further confirms our conclusion that liquid films in fibers and vessels are almost inexistent.

Remarkably, we observe that, although there is no evidence of a connected hydraulic network of free water, (i) the liquid patches in vessels and in fibers disappear simultaneously in the first stage while fibers finish to empty in the second stage; (ii) the drying rate can remain constant while the moisture content decreases to low values (see Figure 3), meaning that sufficient water is continuously transported towards the free surface. Moreover, bound water starts disappearing only when free water (from vessels and fibers) is fully absent.

These different observations suggest that bound water in the solid cell wall structure could play a major role in the drying of the system, by ensuring the connection and transport between the free water patches inside the medium and the free surface of the sample, and then evaporating from the sample.

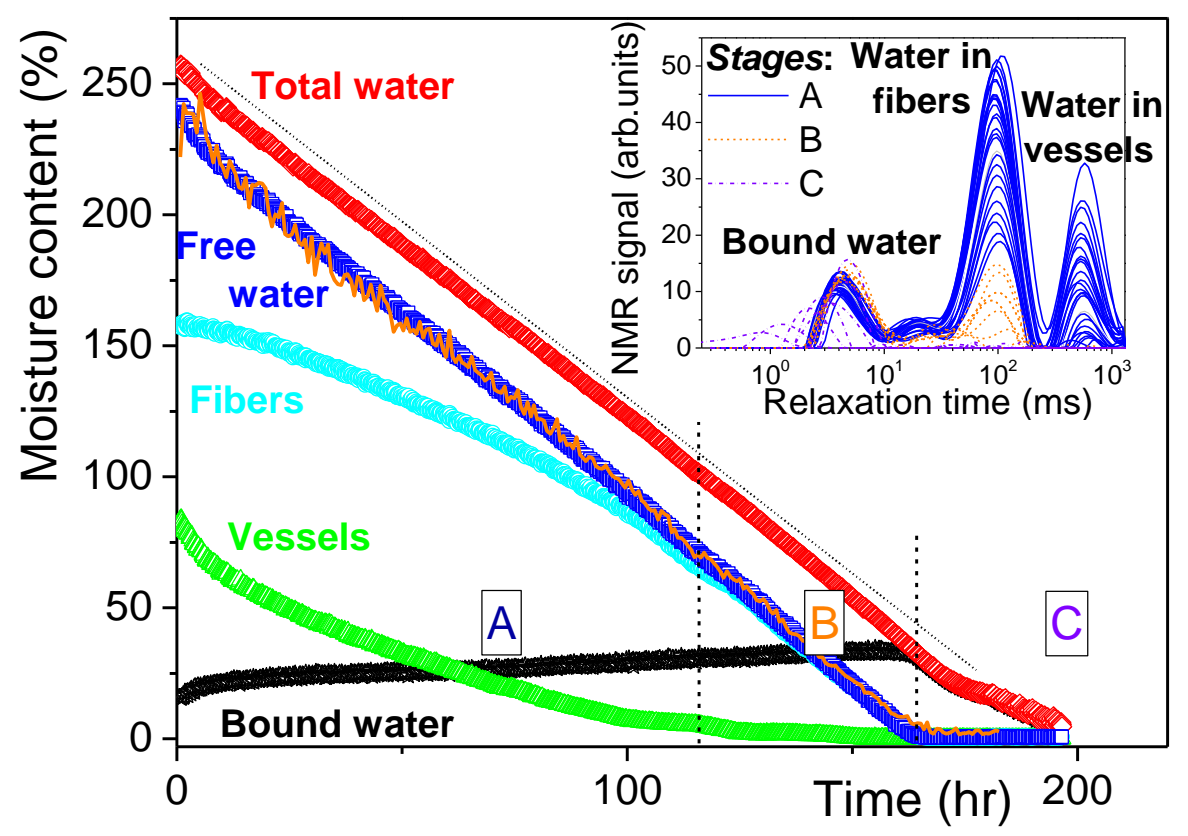

Figure 6: Moisture content of water in the different pore types (free water in vessels or fibers, bound water in cell walls) of a 1-cm-long poplar sample during drying (case (a) of Figure 3) (mass of water in each of this pore type to dry wood mass ratio). The MC of each water class is computed from the integral of the signal in the corresponding region of relaxation time in the distribution shown in the inset. Free water corresponds to the sum of the signal for vessels and fibers. Total water is computed from the sum of the signal for free and bound water. The straight dotted line is a guide for the eye. The orange curve in the main graph corresponds to the integral of $1 \mathrm{D}$ profiles in time (corresponding a priori to free water). The inset shows the distribution of relaxation time at different times during this test (every $9 \mathrm{~h}$ ). Different line types and colors (see legend) are used for the different stages (A, B and C) identified in the main graph and separated by dashed lines. 


\section{Physical origin of bound-water transport}

The potential energy of the system is lower when water molecules are placed between the initially neighbouring polymer molecules composing cell-walls (essentially cellulose and hemicelluloses) which thus tend to absorb water. When there is some gradient of water concentration the molecules start propagating inside the cell-walls to tend to an even distribution of the number of water molecules in the cellulosic structure, which is at the origin of the diffusion process.

The extraction of free water from the wood interior and its transport towards the free surface should occur as a result of an imbalance between the (lower) chemical potential of bound water along the free surface of the sample, in contact with air with $n<1$, and the (larger) chemical potential of liquid water in depth in the sample. Note that inside the sample, owing to the presence of liquid patches and bound water saturating the wall, we can consider that $n=1$ (see above). The chemical potential of the liquid at ambient pressure and temperature is $\mu_{0}$, and that of the vapor is $\mu_{0}+R T \ln (n)$ (see, e.g., [50]). Remarkably, a relative humidity slightly smaller than 1 , even by a value as small as $1 \%$, gives a large chemical potential difference [51], which induces a diffusion of the bound water towards the free surface of the sample through the cell walls.

If the liquid is contained in an airtight pore the pressure in the liquid is strongly decreased, which may induce cavitation. Such a phenomenon was demonstrated to occur [51-54] and properly modeled in the case of pores filled with liquid and dispersed in a hydrogel, which may be considered as model materials of wood $[34,52]$, at least concerning fluid transfer characteristics. It is not clear whether this situation is frequent in wood, as there might also be a large number of very thin air connections throughout the structure when the material is partially saturated. The main result of the above considerations is that a gradient of chemical potential is a powerful means to extract liquid water.

In the case of wood, a complete description of the transport process resulting from the chemical potential drop would require a detailed knowledge of physico-chemical characteristics of the cell walls. Here, in order to roughly quantify the process, we will simply directly consider the resulting diffusion process described by Fick's law with a diffusion coefficient $D$ for the bound water through a wood saturated with bound water. For a homogeneous medium and in steady state, Fick's law tells us that the flux between the liquid source and the free surface (situated at a distance $h$ ) is proportional to $D / h$. For a medium in which the liquid sources are dispersed over the sample height $H$ and partially cover the cell-wall surfaces (coverage described by the fraction $\phi$ ), and the bound water content occupies a volume fraction $\eta$, we then expect a characteristic velocity on the order of $V_{d}=\eta f(\phi) D / H$. In this expression $\eta$ is about $12 \%$ (estimated from the FSP), and $f$ is an unknown function such that $f(1)=1$ and decreasing slowly as $\phi \rightarrow 0$, since a limited number of interfaces between cell walls and liquid is likely sufficient to ensure a maximum flow rate.

The value of $D$ may be found from the similar diffusion process observed when (dry) wood is put in contact with a liquid bath. In that case it was indeed shown that bound water progresses in advance of the free water front [26, 34], which means that it diffuses longitudinally in the cell walls. The bound water progression was well described by considering diffusion from a moving boundary (i.e., the advancing front of free water), which led to estimate the coefficient of diffusion as $D \approx 6 \times 10^{-9} \mathrm{~m}^{2} \mathrm{~s}^{-1}$ [33]. This value is at the same magnitude as those reported before but measured differently [55-58]. Finally we find that the initial (here we assume $\phi=1$ ) water velocity induced by diffusion should be $V_{d} \approx 7 \times 10^{-8} \mathrm{~m} \mathrm{~s}^{-1}$ for $H=1 \mathrm{~cm}$.

\section{Water dynamics at the local scale}

We can get a direct view of this phenomenon of absorption then transport of free water through cell wall from successive 3D XRCT images in time. In Figure 7 we see a vessel containing two large regions (bottom 
and top) filled with air, and the liquid volume between these two regions significantly decreases in time (over a few minutes). Let us search for the origin of this liquid transport. This liquid may evaporate, be transported via liquid film and vessel walls, move to ray or fibers, or be absorbed in cell walls. First of all, as the relative humidity all along the vessel is close to $100 \%$ (see above), evaporation from the liquid-air interface towards the sample free surface would be much too slow as compared to the observed dynamics. Moreover, in any event, it could not explain the growth of the second (bottom) bubble in the channel, as the vapor formed, blocked by liquid patches in the vessel, could not reach the sample free surface. Then, since the contact angles of the liquid-air interfaces are between 0 and $15^{\circ}$ there can hardly be liquid films along cell walls in these regions allowing some transport along the vessels (see demonstration above). We also checked from images in a RT plane that this vessel has no neighboring vessel into which the water could have been driven by capillary force (through connection by pits). We deduce that this liquid volume necessarily flows through the cell walls in the form of bound water. The liquid might also flow towards some nearby fibers or wood rays. This nevertheless seems rather unlikely as this would require a large number of them in order to store such a volume of liquid. Moreover, this stored liquid would then have to escape from these fibers, which are dead-end channels, or rays, which are aligned in a direction perpendicular to the longitudinal direction. Then, the liquid would likely be extracted through their own cell walls, and this would a priori also occur via bound water.

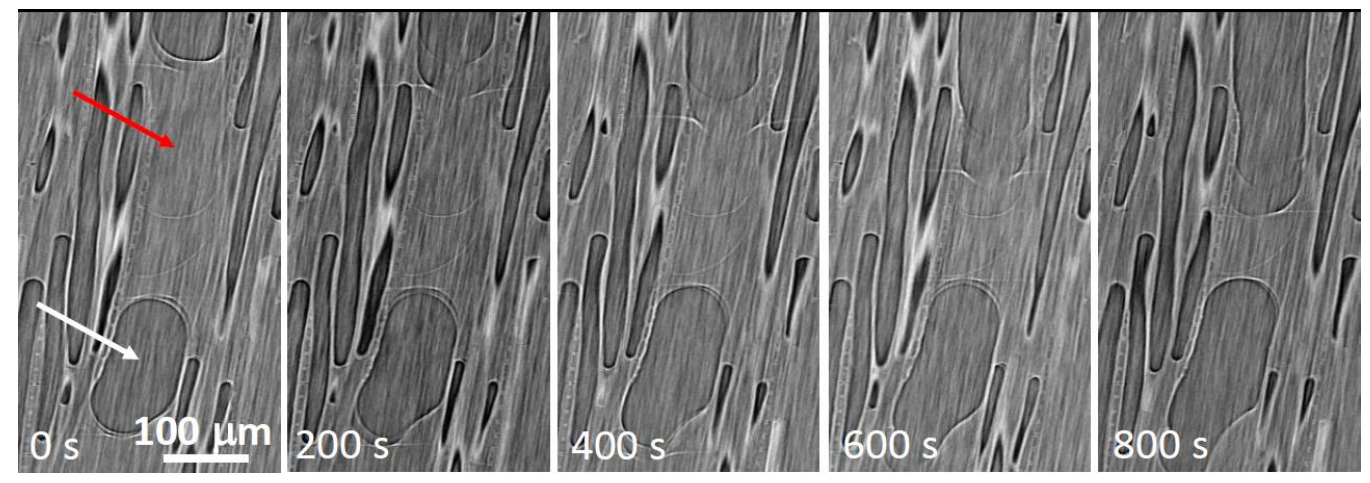

Figure 7: Successive views (every $200 \mathrm{~s}$ ) of the internal structure of a wood sample (sample length: $4 \mathrm{~cm}$ ) at $3.7 \mathrm{~mm}$ (mean distance) below the free surface in a TL plane (see Figure 1) during drying in the constant rate regime ( $M C=151 \%)$. A liquid region in a vessel is shown with the red arrow. An air region (bubble) in the same vessel is shown with the white arrow.

On the other hand, we can observe the growth of the bottom air region (see white arrow) in Figure 7. This shows that some air is available somewhere in the wood and is attracted there or pushed. Since air is perfectly wetting the liquid and solid surfaces it can easily move from one place to another through very small pores, driven by small pressure differences, but this necessarily induces liquid water motion. A similar, more spectacular effect is shown in Figure 8 (red arrow) where we can also observe the sudden birth of a bubble inside a liquid water region. Such an effect is reminiscent of cavitation. However, standard cavitation cannot occur in a vessel open to the sample free surface at short distance and subjected to the ambient pressure and bubble creation via radiation damage was not observed in the whole volume of this time series. More likely, this bubble forms as a result of the complex transports of liquid in the porous structure and the corresponding pressure distribution evolution. 

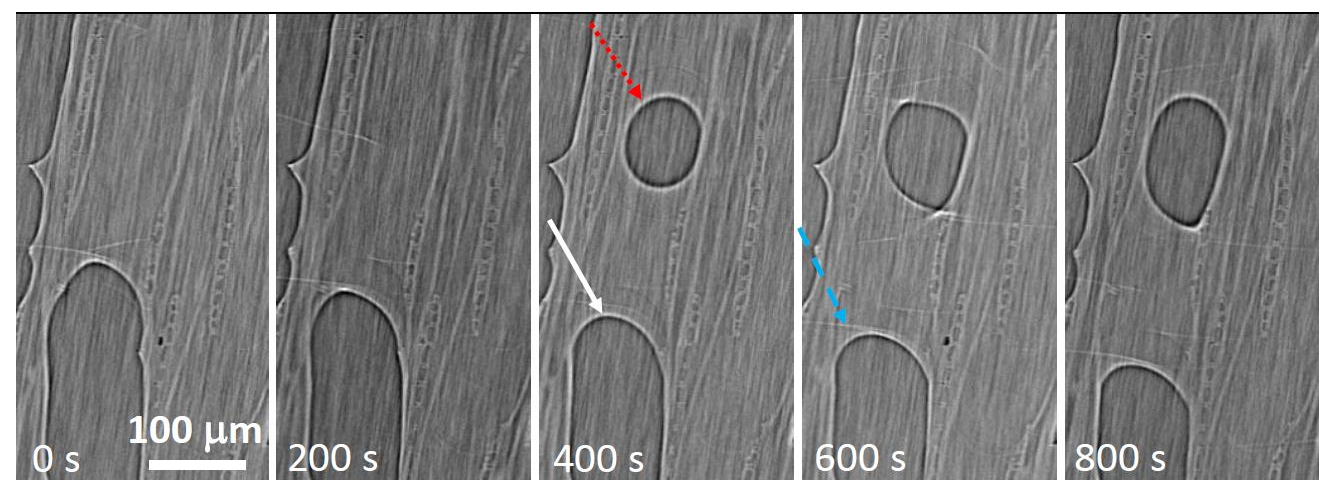

Figure 8: Successive views (every $200 \mathrm{~s}$ ) of the internal structure of a wood sample (sample length: $4 \mathrm{~cm}$ ) at $3.7 \mathrm{~mm}$ (mean distance) below the free surface in a TL plane (see Figure 2) during drying in the constant rate regime $(M C=151 \%)$. A bubble (see red dotted arrow) appears on the third picture and then grows; this apparently pushes downwards the liquid and the bubble (see white arrow) situated below the bubble. Note that artefacts appear as light white lines due to liquid motion during the time of acquisition (see blue dashed arrow).

Considering now the situation inside the fibers we also expect the diffusion of water through the cell walls towards the free surface. If the fibers were perfectly airtight, cavitation should occur (see above). Nevertheless, there might also exist extremely thin paths allowing air to be transferred through fiber walls and precluding cavitation. In that case air and liquid transfers in fibers could play a role in the pressure redistribution process mentioned above.

\section{$X$. Non-linear evolution of the processes at a local scale}

We thus have a water transport basically ensured by chemical potential imbalance. In a wood sample, the solid network which ensures the bound-water transport is complex, with walls with various concentrations and connections between them or with the liquid in pores. Even if, on average, during drying, the chemical potential at the sample free surface is lower than inside the sample, the detailed distribution of chemical potentials in this network is obviously quite complex. Moreover, as the distribution of liquid and air in the porous structure continuously evolves, so does the distribution of chemical potentials. As a result, we expect a somewhat chaotic process of liquid extraction throughout the medium. This is effectively illustrated by the liquid patch distribution observed in a cross section of the sample (see Figure 9). We may first see that some filled fibers form alignments, an effect which might be due to the proximity of rays along this line. Here we will not discuss further this aspect and rather consider the following critical points:

(i) some vessels and fibers empty but, without simple or systematic rule of localization of these events, e.g., some fibers may empty while they are close to filled vessels;

(ii) a significant fraction of fibers, and even some vessels, fill during drying; this filling does not follow any simple rule either, e.g., some fibers fill although they are surrounded by dry fibers.

In this context, the wide range of contact angles observed between liquid drops and cell walls would be due to the complex distribution of bound water concentration, and the critical impact of this concentration on wetting properties of wood [34]. 


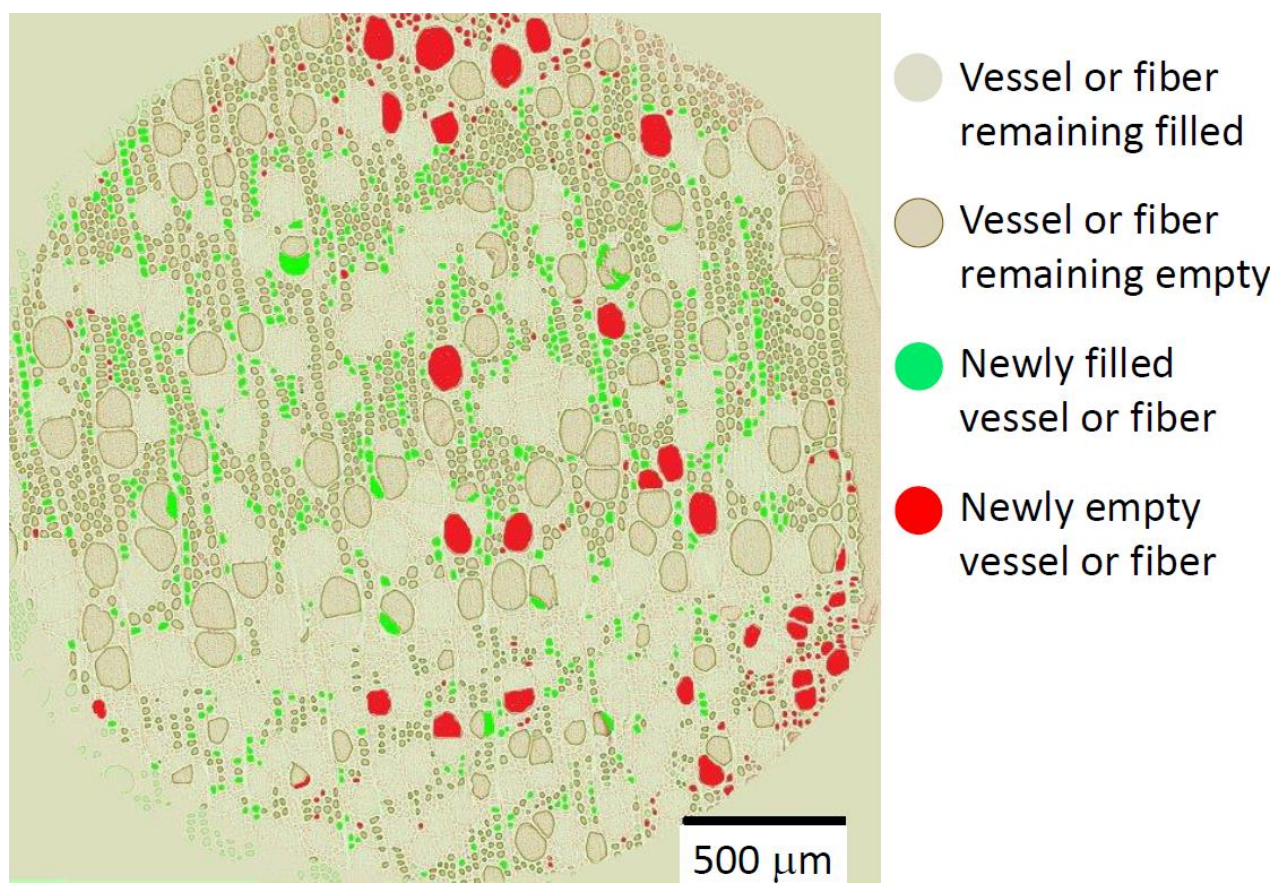

Figure 9: Evolution of the liquid distribution in a wood sample (sample length: $4 \mathrm{~cm}$ ) at $3.7 \mathrm{~mm}$ (mean distance) below the free surface in a RT plane (see Figure 2) during drying in the constantrate regime. The evolution of water content in fibers and in vessels when the moisture content evolves from $M C=170 \%$ to $M C=151 \%$ is represented by different colors (see legend).

\section{Drying scheme for wood}

The following scheme of water transport during slow drying emerges from the NMR and XRCT results (see Figure 10):

- Most of the evaporation occurs from bound water at the sample free surface; evaporation of bound water from the cell walls inside the sample can also occur but since the RH inside the sample is close to 1 , the resulting vapor transport by diffusion from these surfaces remains negligible (as in the case of a channel whose internal surfaces are covered with liquid [49]);

- As long as there is free water in the sample the evaporated bound water is replaced by bound water formed by absorption of free water in the cell walls;

- When there is no more free water the drying goes on by progressively decreasing the bound water content in the sample

Note that this scheme in particular provides a straightforward explanation to the effect of homogeneous desaturation down to complete disappearance of free water for a very slow drying (case (a) in Figures 3 and 6). 


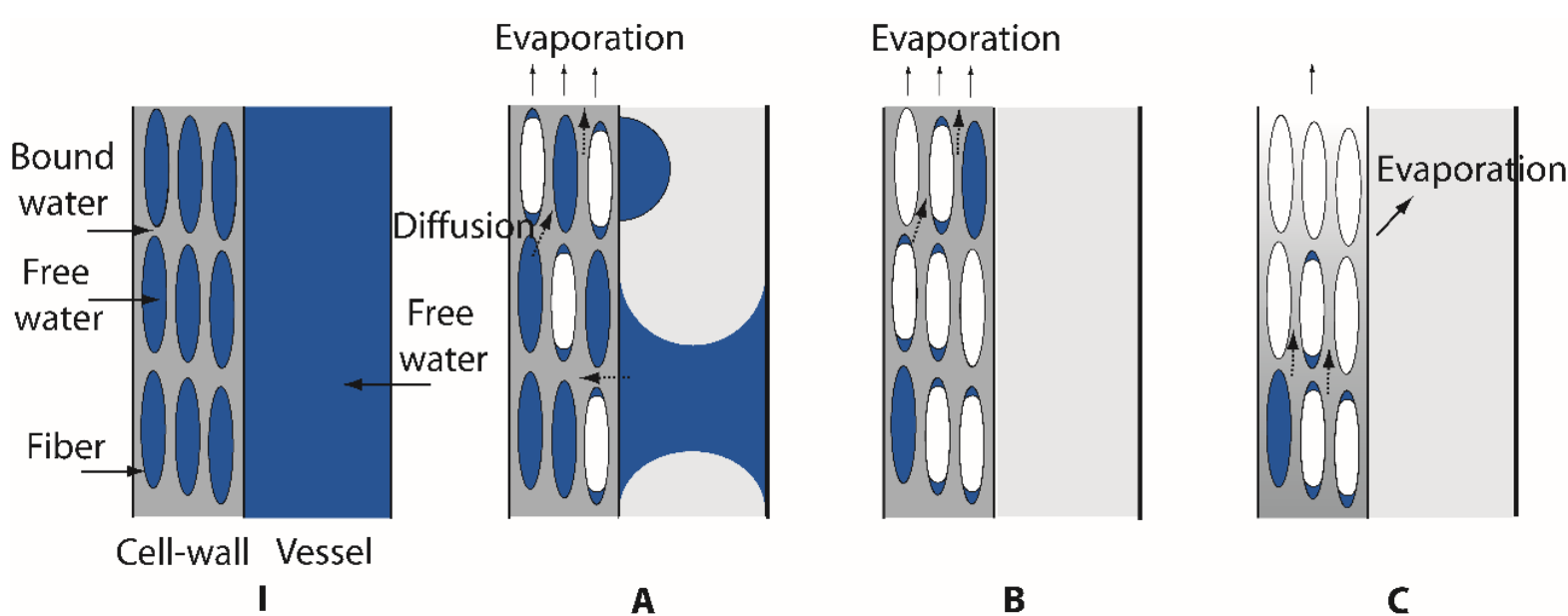

Figure 10: Scheme of principle of water transport at the different stages of (longitudinal) drying of wood: (I) Initial stage, (A) free-water extraction from fiber and vessel towards cell walls then diffusion towards free surface, (B) vessels are empty - extraction of free water from fibers then diffusion, (C) vessels and fibers are empty over some distance below the free surface - extraction of bound water leading to the formation of a concentration gradient in this region - main evaporation from around the upper boundary of free water. Note that the drying rate is constant in stages $A$ and $B$, and decreases in stage $C$. These regimes correspond, for example, to those identified in Figure 6 . Note that the remaining fraction of free water in fibers in regime $C$ depends on the drying rate. For sufficiently slow drying there is no more water in fibers in this regime, as in the case of Figure 6; for large drying rate and/or large sample length, some water still exists in fibers in depth as in the case of Figure 11; for even larger drying rate and/or sample length, there might still exist some water in vessels, as in Figure 12.

\section{Water extraction dynamics controlled by bound water}

A constant-rate period is observed for sufficiently slow imposed air flow and/or sufficiently large water content in the sample (see Figure 3). Moreover, the drying rate is constant as long as free water exists over the whole length of the sample, and starts to decrease when a region without free water starts developing from the sample top (see insets of Figure 3 and red disks). Since we have seen that the free water is transported as bound water towards the free surface, these observations suggest that there are some critical conditions in terms of evaporation along the free surface and/or free-water concentration for which the freewater transport is not sufficiently fast to feed the top of the sample. Since this free-water transport is ensured by diffusion of bound water in cell walls its rate is given by the characteristic diffusion velocity $V_{d} \approx 7 \times 10^{-8} \mathrm{~m} \mathrm{~s}^{-1}$ which here appears to be larger or of the same order as the drying rate in the constantrate period for cases (a) and (b) (respectively $V \approx 1.3 \times 10^{-8} \mathrm{~m} \mathrm{~s}^{-1}$ and $V \approx 6 \times 10^{-8} \mathrm{~m} \mathrm{~s}^{-1}$ ) (see Figure 3). In those cases, the diffusion through cell walls is sufficiently fast to extract the free water from the sample bottom and continuously supply bound water for evaporation along the sample free surface. This is also consistent with the observation that the free-water content profiles are uniform (see insets (a) and (b) of Figure 3), i.e., the water content transport by diffusion in cell walls is sufficiently fast to reach an equilibrium throughout the medium. In case (c) the initial drying rate $\left(8.5 \times 10^{-8} \mathrm{~m} \mathrm{~s}^{-1}\right)$ is larger than $V_{d}$, which explains that the water-content profiles do not remain uniform (see inset (c) in Figure 3), more water is extracted from the top layer of the sample, i.e., the diffusion of bound water is too slow with regards to the drying rate to ensure a complete re-equilibration of the free water distribution. Note that in various other more or less complex systems containing water in different states, the water distribution remains homogeneous for sufficiently slow drying: bead packings [46], emulsions [59], soils [60], packings of hydrogel beads [61], etc. The case of hydrogel particle packing is more interesting as the water has to diffuse through the particles, and between 
them, to reach the sample free surface, as in wood cell walls. A similar criterion then arises [61] for describing the different regimes, quantified by the Biot number, which describes the competition between drying rate and the rate at which water diffuses and homogenizes the moisture content. However, for this material there is no coexistence of free and bound water, with possible coupling of their transport, as in wood.

In this case (c), the drying characteristics are globally similar to those identified above, i.e., extraction of water from fibers and vessels in a first stage, then full emptying of fibers followed by bound-water extraction (see Figure 11). It is worth noticing that the drying rate remains approximately constant despite the existence of a gradient of water content (see case (c) in Figure 3), as long as the concentration of free water is not zero close to the free surface of the sample. This suggests that the liquid transport is still ensured up to the free surface and supplied for evaporation at the imposed rate. The situation significantly changes when the freewater content reaches zero at the approach of the sample free surface: the drying rate starts to decrease significantly (see Figure 3). During this drying rate decrease free water is still significantly removed from the sample, but an important difference is that this also (approximately) corresponds to the beginning of a bound-water content decrease (see Figure 11, transition from regime $\mathrm{B}$ to regime $\mathrm{C}$ ). Moreover, during this period, the peak of $T_{2}$ associated to bound water significantly spreads (see inset of Figure 11), instead of just decreasing in amplitude as for free water or being essentially shifted towards lower $T_{2}$ value like in case (a) (see inset of Figure 6). This means that the decrease of bound-water amount in the sample is heterogeneous, i.e., a gradient of water concentration in the bound state develops. Obviously, since the water is extracted from the sample from one face, we expect this gradient to develop mainly at the approach of the sample free surface.

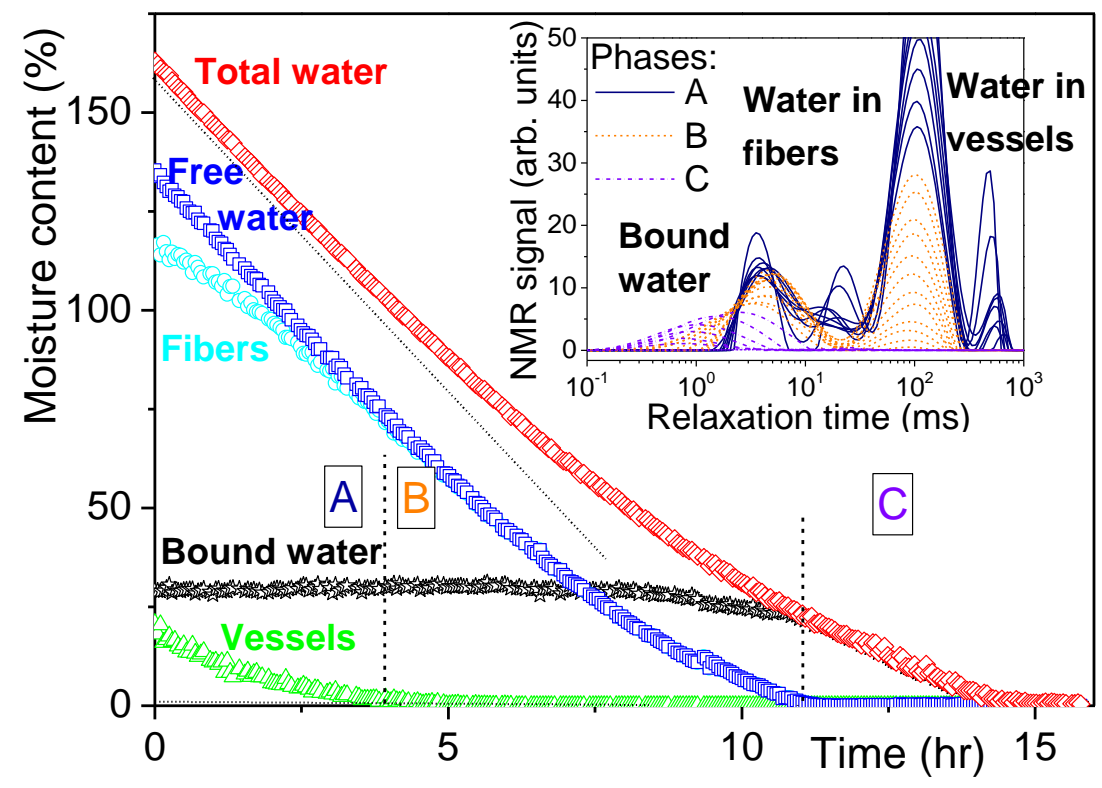

Figure 11: Moisture content of water in the different pore types of a 1-cm-long poplar sample during drying (case (c) of Figure 3 ) (mass of water in this pore type to dry wood mass ratio). The straight dotted line is a guide for the eye. The inset shows the distribution of relaxation time at different times during this test (every $0.5 \mathrm{~h}$ ). Different line types and colors (see legend) are used for the different stages (A, B and C) identified in the main graph and separated by dashed lines. 
In order to gain further insight on the process in the non-constant drying rate regime we carried out a test on a longer sample, which makes it possible to follow the deformations too, and followed its characteristics by MRI (see Section III). Here, as the sample length is $4 \mathrm{~cm}$, we have $V_{d} \approx 2 \times 10^{-8} \mathrm{~m} \mathrm{~s}^{-1}$, which is much smaller than the initial drying rate (see Figure 12). As a consequence, we expect a heterogeneous drying, since the free water transport will not be sufficiently fast to supply water at the imposed rate of evaporation at the free surface. We nevertheless clearly observe a first stage during which the drying rate is constant (see inset (a) in Figure 12) as long as the free water profile, although non-uniform, extends up to the free surface (see inset (b) in Figure 12). Then the drying rate continuously strongly decreases as the moisture content decreases, and a region without free water grows.

We can appreciate the bound-water distribution from deformation measurements, since we know that wood strains are basically associated with bound-water content [33]. However, we will not elaborate further on this point and just consider our measurements as qualitative information on the bound-water distribution. Remarkably, the bound water distribution exhibits a plateau in the region where there is still free water and, beyond that region, decreases towards the free surface (see inset (b) of Figure 12). This confirms that a bound water gradient develops when no more free water is available, which leads to the decrease of the drying rate. Actually, this gradient could allow the transport of bound water towards the free surface, but with a characteristic velocity of the same order as $V_{d}$, which is not sufficient. As a consequence, we expect that this transport is negligible and the top layers progressively fully dry, forming a dry front.

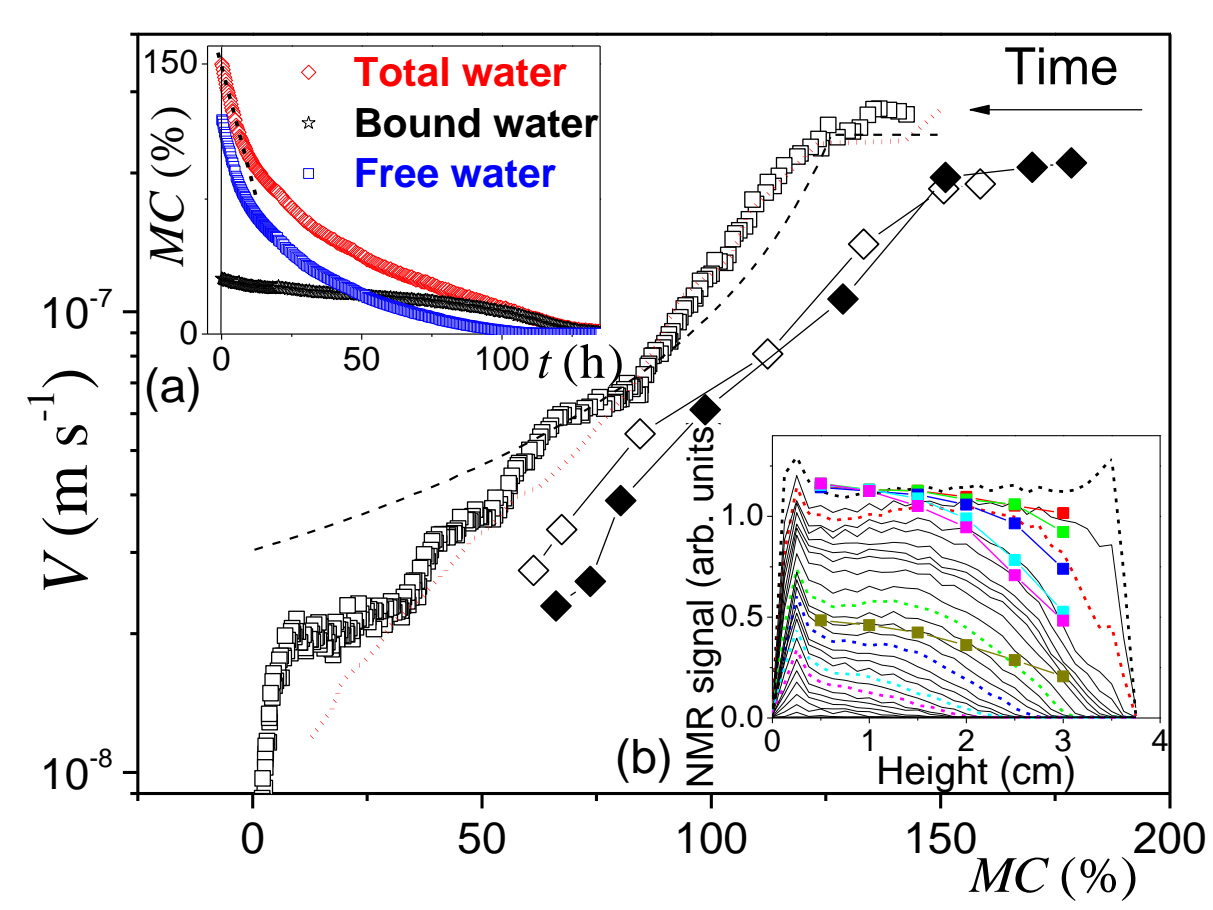

Figure 12: Drying of a long wood sample ( $4 \mathrm{~cm}$ along L-axis) in the MRI set up, subjected to a dry air flow: drying rate as a function of moisture content (squares). The red dotted line represents the model prediction from dry front thickness measured from MRI profiles (see text). The black dashed line corresponds to the model of Section XII. The filled diamonds correspond to a (green wood) sample dried under ambient conditions ( $\mathrm{RH}$ around $50 \%, 22^{\circ} \mathrm{C}$ ) and data obtained from mass measurements during XRCT experiments. The open diamonds correspond to a drying under the same conditions of a poplar sample re-soaked after initial drying. The top left inset shows the evolution of the two main water types in time. The bottom right inset shows 1D free water 
profiles (lines) at different times: (from top to bottom) 0, 2, 4.5, 7, 9, 12, 14, 16, $20 \mathrm{~h}$ and then every $5 \mathrm{~h}$. The filled squares correspond to deformation profiles at (from top to bottom) 0 and 4.5 (red), 30, 50, 70, 80, $140 \mathrm{~h}$. The corresponding free water profiles are represented in the same colored dashed line.

Under these conditions, another transport process is necessary to explain the removal of free water from the sample. In the absence of possible transport as bound or free water along the upper region, we must turn to a transport in the vapor state. More precisely, we can consider that most vapor forms around the highest free water-air interfaces in the sample, and then diffuses along the upper region (which does not contain free water). Such a process is similar to that observed in granular packings in the so-called "Falling Rate Period" [46, 62], and can be described with the help of the above approach providing the drying rate as $V=\rho_{0} D_{v} / \rho\left(h+\delta^{*}\right)=V_{0} /\left(1+h / \delta^{*}\right)$ with $\delta^{*}=D_{v} \delta / D_{0}$. Here, from MRI data (see inset (b) of Figure 12) we can estimate the distance $(h)$ of the region without free water and fit the drying rate to the observed one by choosing an appropriate value for $D_{v} / D_{0}$, i.e., the sole fitting parameter. It appears that the prediction (red dotted line in Figure 12) of this model is remarkable since it represents the drying rate evolution very well over almost the whole range of moisture content. The value of 0.55 obtained for $D_{v} / D_{0}$ is nevertheless slightly larger than the value we could expect if we considered that the vapor has to be transported mainly along the vessels, for which we have $\tau \approx 1$ and $\varepsilon \approx 0.23$, leading to $D_{v} / D_{0} \approx 0.23$. This suggests that we identified the basic process correctly but there might be some amplification of the diffusion due to the presence of the bound water in cell walls, providing an additional source of evaporation along the vessel walls. Also note that the above approach neglects the extraction of bound water from the free surface, which in that case continues all along the process.

We can remark that the free water is still transported in the deeper layer through bound water. Indeed, a pure motion due to water vapor would induce a growing dry region without water content variations below the front, whereas here we have a further homogeneous decrease of the water content in the saturated bound water region below the front (see inset b of Figure 12). A similar process resulting from capillary effects was shown to occur below the dry front in the "Falling Rate Period" with bead packings [46]. Here this effect is again the hallmark of an equilibration process in this region, which logically again occurs thanks to freewater transport by bound water diffusion, which has now more time to act in such a shorter region and under a lower drying rate.

Finally, with some approximations, we can propose the following model for describing the drying rate of a wood sample along the longitudinal $L$ direction, i.e. when the air flux is in a transversal RT plane. We consider the initial drying rate $V_{0}$ imposed by external conditions (air flux, temperature, $\mathrm{RH}$ ) along a water surface of the same shape as the wood RT cross-section. We also note that the characteristic velocity of diffusion of bound water expresses as $V_{d}=\eta f(\phi) D / H$, in which the concentration $\phi$ is related to the moisture content MC. The remaining unknown here is the function $f$.

1) As long as $V_{0}<V_{d}, M C>M C_{c}$ with $M C_{c}$ such that $V_{0}=\eta f\left(M C_{c}\right) D / H$, the drying rate is constant and equal to $V=V_{0}$

2) For $V_{0}<V_{d}, M C<M C_{c}$ and the drying rate may be approximated by considering that a dry region develops from the sample top, which leads to an expression similar to equation (5): $V=V_{0}\left[1+H\left(M C_{c}-M C\right) \rho V_{0} / 2.5 \varepsilon \rho_{0} D_{0} M C_{c}\right]^{-1}$.

The prediction of this model is compared with our observations for the test in Figure 12, showing a relatively good agreement except at very low moisture content. 


\section{Conclusion}

We have shown that during wood drying, even well above the Fiber Saturation Point, bound water diffusion in cell walls ensures the extraction of liquid water from pores and its transport towards the surface of evaporation, and thus controls the drying rate. The distribution of bound water content (uniform or heterogeneous) along the main sample axis and the drying rate evolution depend on the competition between the external conditions and a characteristic rate of transport due to bound water diffusion. These results show that the drying of a hygroscopic material with pores filled with liquid is controlled by the bound phase, which opens the way to the understanding or control of the properties of many other materials containing both water types in food or civil engineering applications. Also note that no differences on the drying dynamics (see Figure 12) or on the qualitative characteristics of the liquid distribution inside the sample were observed between green wood and re-soaked wood samples. Thus it appears that the drying mechanisms do not differ for both systems.

In this context, we can remark that NMR techniques, and in particular relaxometry in time (or "dynamic relaxometry"), appears to be a powerful technique to observe the transport of different types of water and the evolution of the filling of the different types of pores. It should bring a lot in the future concerning the transport in other wood types and/or during drying in other directions.

Note that here we only considered drying along the longitudinal direction as this is the fastest, but we expect that the mechanisms observed in this case will also apply for drying in transversal or radial directions, i.e., drying controlled by bound water diffusion allowing first the extraction of free water then bound water. The transition from the first regime associated with constant drying rate to the second regime governed by diffusion might nevertheless occur sooner than in the longitudinal direction as the diffusion coefficient is smaller [63], and, consequently, the rate of drying in this second regime will be smaller.

Concerning plants, it is remarkable to see that bound water diffusion, which was proved to control imbibition in hardwood rather than capillarity [33-34], also controls the opposite process of drying. This suggests that the transfers between bound and free water might play a major role in different aspects of the functioning of plant systems, and in particular in the debated problem of the refilling of cavities after embolism [64-69] or in the problem of maintenance of whole-plant water balance thanks to transfers between compartments [69].

\section{Acknowledgements}

This work has benefited from a French government grant managed by ANR within the frame of the national program Investments for the Future ANR-11-LABX-022-01 and has been also supported by a program of the CNRS (Peps INSIS CNRS 'Greenwood'). ANATOMIX is an Equipment of Excellence (EQUIPEX) funded by the Investments for the Future program, project NanoimagesX (Grant ANR-11-EQPX-0031). The authors express their gratitude to Michel Bornert, Dang-Mao Nguyen, Patrick Aimedieu, Nicolas Ducoulombier and Leila Rostom for their help during image acquisitions at Synchrotron SOLEIL, David Hautemayou and Cédric Mézière for their technical support for the device of the XRCT experiments, and Benoit Coasne, Laurent Brochard, Patrick Huber and Michele Holbrook for fruitful discussions.

\section{Appendix A. Contact angle measurements}

Here we illustrate the measurement technique for estimating the contact angle of liquid-air interfaces with walls inside wood samples from the XRCT images (see Figure 13). 

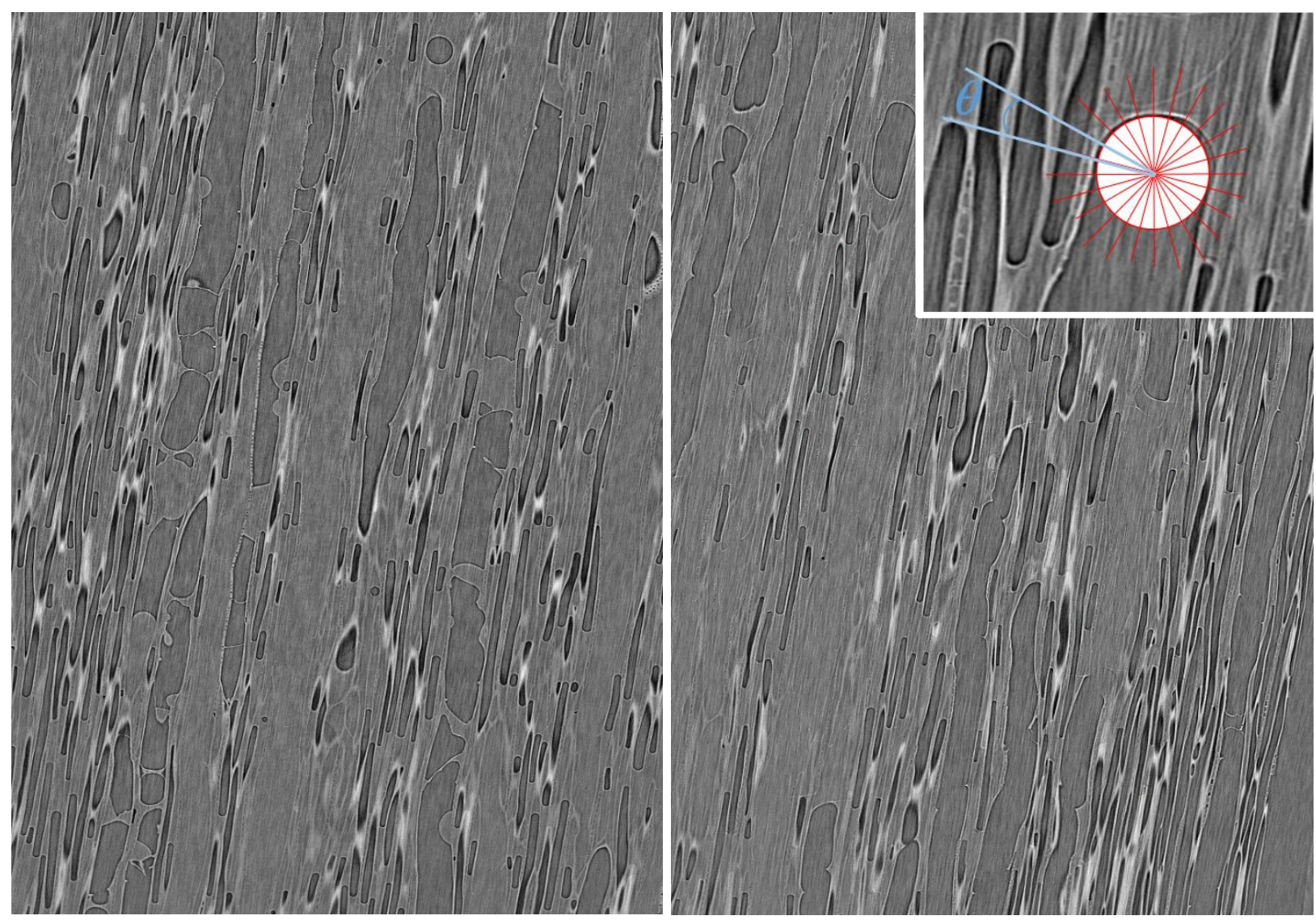

Figure 13: Views of the internal structure of a wood sample (sample length: $4 \mathrm{~cm}$ ) at $3.7 \mathrm{~mm}$ (mean distance) in a TL plane below the free surface during drying in the constant rate regime ( $M C=151 \%$ ). The inset shows a zoom on vessel with the technique for estimating the apparent contact angle (between the two blue lines).

\section{Appendix B. Test under slow drying conditions (case (a)) with a different initial MC}

We present here (see Figure 14) the results of the NMR measurements for the test shown in Figure 3 (blue crosses). 


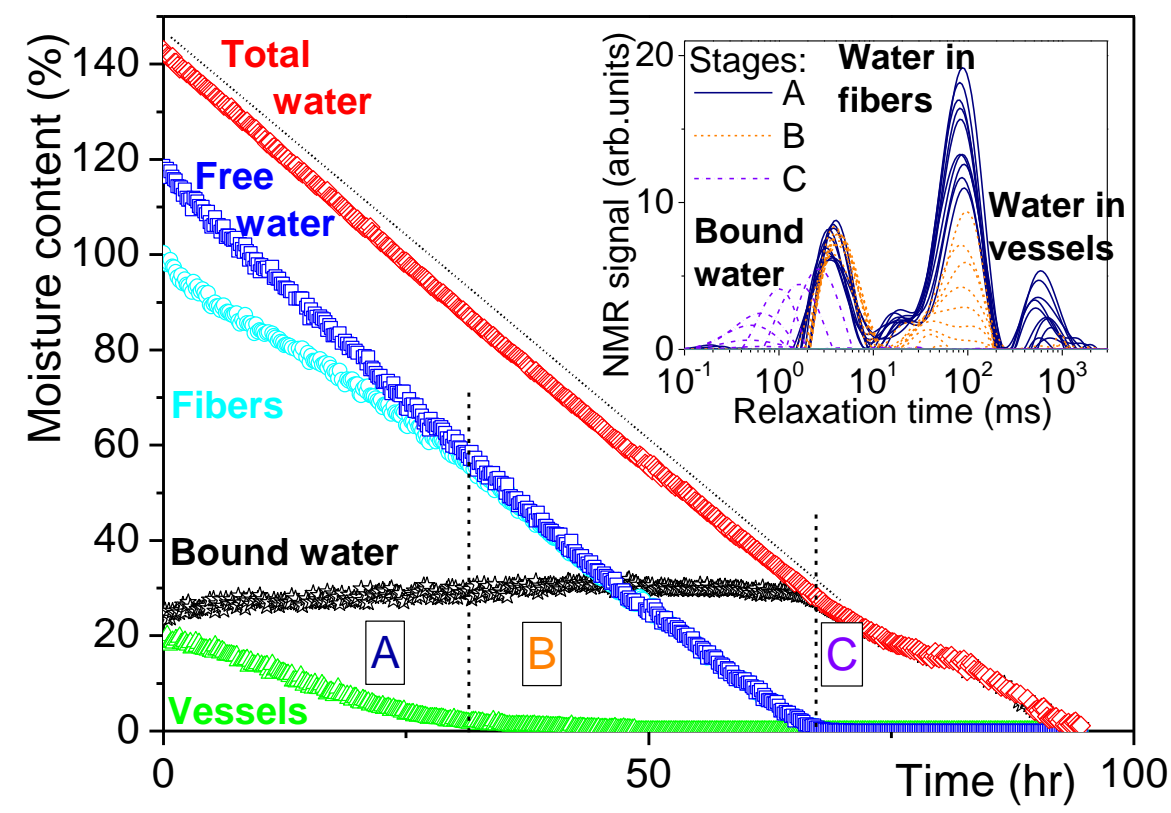

Figure 14: Moisture content of water in the different pore types (free water in vessels or fibers, bound water in cell walls) of a 1-cm-long poplar sample during drying (cross symbols in Figure 3 ) (mass of water in each of this pore type to dry wood mass ratio). The straight dotted line is a guide for the eye. The inset shows the distribution of relaxation time at different times during this test (every $3 \mathrm{~h}$ ). Different line types and colors (see legend) are used for the different stages ( $A, B$ and $C$ ) identified in the main graph and separated by dashed lines.

\section{References}

[1] J. Van Brakel, Mass transfer in convective drying, Adv. Drying, 1, 217-267 (1980)

[2] M. Prat, Percolation model of drying under isothermal conditions in porous media, Int. J. Multiphase Flow, 19, 691-704 (1993)

[3] P. Coussot, P., Scaling approach of the convective drying of a porous medium, European Physical Journal B 15, 557-566 (2000)

[4] N. Shahidzadeh-Bonn, A. Azouni, P. Coussot, Drying rate controlled by wettability in model porous media, Journal of Physics: Condensed Matter, 19, 112101 (2007)

[5] I. N. Tsimpanogiannis, Y. C. Yortsos, S. Poulou, N. Kanepoullos, A.K. Stubos, Scaling theory of drying in porous media, Physical Review E, vol. 59, 4353-4365 (1999)

[6] P. Lehmann, S. Assouline and D. Or, Characteristic lengths affecting evaporative drying of porous media, Physical Review E, 77, 056309 (2008)

[7] N. Shokri, P. Lehmann and D. Or, Characteristics of evaporation from partially wettable porous media, Water Resources Research, 45, 02415 (2009)

[8] L.F. Hawley, Wood-liquid relations, Technical Bulletin, US Dept. Agriculture, Washington, 1-35 (1931)

[9] J. Siau, Transport process in wood (Springer-Verlag, New York, 1984).

[10] J.C.F. Walker, Primary Wood Processing - Principles and practice, Springer, New York, 2006 
[11] A.J. Stamm, Movements of fluids in wood - Part 1: Flow of fluids in wood, Wood Science and Technology, 1, 122-141 (1967)

[12] A.J. Stamm, Movements of fluids in wood - Part 2: Diffusion, Wood Science and Technology, 1, 205-230 (1967)

[13] G. Scheepers, T. Moren, T. Rypstra, Liquid water flow in pinus radiata during drying, Holz Roh Werkst, 65, 275-283 (2007)

[14] S. Sandoval-Torres, A. Perez-Santiago, E. Hernandez-Bautista, Drying model for softwood and moisture patterns measured by magnetic resonance imaging, Drying Technology, 37, 458-467 (2018)

[15] J.G Salin, Drying of liquid water in wood as influenced by the capillary fiber network, Drying Technology, 26, 560-567 (2008)

[16] C. Skaar, Wood-water relations, Springer, Berlin, 1988

[17] R.B. Keey, T.A.G. Langrish, J.C.F. Walker, Kiln-drying of lumber, Springer, Berlin, 2000

[18] O.A. Plumb, G.A. Spolek, B.A. Olmstead, Heat and mass transfer in wood during drying, Int. J. Heat Mass Transfer, 28, 1669-1678 (1985)

[19] M.A. Stanish, G.S. Schajer, F. Kayihan, A mathematical model of drying for hygroscopic porous media, AIChE J., 32, 1301-1311 (1986)

[20] P. Chen, D.C.T. Pei, A mathematical model of drying processes, Int. J. Heat Mass Transfer, 32, 297-310 (1989)

[21] P. Perré, I.W. Turner, A 3-D version of Transpore: a comprehensive heat and mass transfer computational model for simulating the drying of porous media, Int. J. Heat Mass Transfer, 42, 4501-4521 (1999)

[22] A.L. Redman, H. Bailleres, P. Perré, E. Carr, I. Turner, A relevant and robust vacuum-drying model applied to hardwoods, Wood Sci. Technol., 51, 701-719 (2017)

[23] J.G. Salin, Drying of sapwood analyzed as an invasion percolation process, Maderas. Ciencia y tecnologia, 8, 149-158 (2006)

[24] P. Wiberg, T.J. Moren, Moisture flux determination in wood druing drying above fibre saturation point using CT-scanning and digital image processing, Holz als Roh- und Werkstoff, 57, 137-144 (1999)

[25] A. Rosenkilde, J.P. Gorce, A. Barry, Measurement of moisture content profiles during drying of Scots pine using magnetic resonance imaging, Holzforschung, 58, 138-142 (2004)

[26] Ö. Gezici-Koç, S.J.F. Erich, H.P. Huinink, L.G.J. van der Ven, O.C.G. Adan, Bound and free water distribution in wood during water uptake and drying as measured by 1D magnetic resonance imaging, Cellulose, 24, 535-553 (2017)

[27] X. Li, X. Wang, M. Zhang, An investigation of the drying rate of water in wood at different relative humidities studied by time domain nuclear magnetic resonance, BioResources, 12, 2991-3000 (2017)

[28] Z. Wang, X.M. Wang, Z.J. Chen, Water states and migration in Xinjiang poplar and Mongolian Scotch pine monitored by TD-NMR during drying, Holzforschung, 72, 113-123 (2018)

[29] X. Li, Y. Gao, M. Zhang, X. Wang, X. Wei, Water migration in poplar wood during microwave drying studied by time domain nuclear magnetic resonance (TD-NMR), Holzforschung, 71, 881-887 (2017)

[30] Md.I.H. Khan, R.M. Wellard, S.A. Nagy, M.U.H. Joardder, M.A. Karim, Experimental investigation of bound and free water transport process during drying of hygroscopic food material, Int. J. Thermal Sciences, 117, 266-273 (2017)

[31] T. Defraeye, P. Verboven, Convective drying of fruit: role and impact of moisture transport properties in modelling, Journal of Food Engineering, 193, 95-107 (2017)

[32] R.H. Nosrati, U. Berardi, Hygrothermal characteristics of aerogel-enhanced insulating materials under different humidity and temperature conditions, Energy and Buildings, 158, 698-711 (2018)

[33] M. Zhou, S. Caré, D. Courtier-Murias, P. Faure, S. Rodts, P. Coussot, Magnetic resonance imaging evidences of the impact of water sorption on hardwood capillary imbibition dynamics, Wood Science and Technology, 52, 929-955 (2018) 
[34] M. Zhou, S. Caré, A. King, D. Courtier-Murias, S. Rodts, G. Gerber, P. Aimedieu, M. Bonnet, M. Bornert, P. Coussot, Liquid uptake governed by water adsorption in hygroscopic plant-like materials, Physical Review Research, 1, 033190 (2019)

[35] K.P. Whittall, A.L. MacKay, Quantitative interpretation of NMR relaxation data, J. Magn. Reson. 84, 134152 (1989)

[36] S.W. Provencher, A constrained regularization method for inverting data represented by linear algebraic or integral equations, Comput. Phys. Commun., 27, 213-227 (1982)

[37] P. Faure, S. Rodts, Proton NMR relaxation as a probe for setting cement pastes, Magn. Reson. Imaging, 26, 1183-1196 (2008)

[38] S. Philippot, J.P. Korb, D. Petit, H. Zanni, Analysis of microporosity and setting of reactive powder concrete by proton nuclear relaxation, Magn. Reson. Imaging, 16, 515-519 (1998)

[39] K.R. Brownstein, C.E. Tarr, Spin-Lattice relaxation in a system governed by diffusion, J. Magn. Reson., 26, 17-24 (1977)

[40] T. Lerouge, B. Maillet, D. Courtier-Murias, D. Grande, B. Le Droumaguet, O. Pitois, P. Coussot, Drying of a compressible biporous material, Phys. Rev. Applied, 13, 044061 (2020)

[41] T. Weitkamp, M. Scheel, J.-L. Giorgetta, V. Joyet, V. Le Roux, G. Cauchon, T. Moreno, F. Polack, A. Thompson, and J.-P. Samama, The tomography beamline ANATOMIX at Synchrotron SOLEIL, J. Phys. Conf. Series 849 (2017) 012037, doi:10.1088/1742-6596/849/1/012037

[42] A. Mirone, E. Brun, E. Gouillart, P. Tafforeau, J. Kieffer, The PyHST2 hybrid distributed code for high speed tomographic reconstruction with iterative reconstruction and a priori knowledge capabilities, Nucl. Instrum. Methods Phys. Res. B, 324, 41-48 (2014)

[43] C.T. Rueden, J. Schindelin, M.C. Hiner, B.E. DeZonia, A.E. Walter, E.T. Arena, K.W. Eliceiri, ImageJ2: ImageJ for the next generation of scientific image data. BMC Bioinformatics 18, 529 (2017)

[44] M. Suzuki, S. Maeda, On the mechanism of drying of granular beds, J. Chem. Eng. Jpn, 1, 26 (1968)

[45] J.F. Daian, Equilibrium and transfers in porous media (Wiley, New York, 2014)

[46] J. Thiery, S. Rodts, D.A. Weitz, P. Coussot, Drying regimes in homogeneous porous media from macroto nanoscale, Phys. Rev. Fluids, 2, 074201 (2017)

[47] P. Lehmann, D. Or, Effect of wetness patchiness on evaporation dynamics from drying porous surfaces, Water Resources Research, 49, 8250 (2013)

[48] F. Chauvet, P. Duru, S. Geoffroy and M. Prat, Three periods of drying of a single square capillary tube, Physical Review Letters, vol. 103, 1-4, 2009.

[49] E. Keita, S.A. Koehler, P. Faure, D.A. Weitz, P. Coussot, Drying kinetics driven by the shape of the air/water interface in a capillary channel, Eur. Phys. J. E, 39:23 (2016)

[50] O. Coussy, Mechanics and physics of porous solids, (Wiley, New York, 2010)

[51] T.D. Wheeler, A.D. Stroock, Stability limit of liquid water in metastable equilibrium with subsaturated vapors, Langmuir, 25, 7609-7622 (2009)

[52] T.D. Wheeler, A.D. Stroock, The transpiration of water at negative pressures in a synthetic tree. Nature 455, 208-212 (2008).

[53] O. Vincent, P. Marmottant, P.A. Qinto-Su, and C.D. Ohl, Birth and growth of cavitation bubbles within water under tension confined in a simple synthetic tree. Phys. Rev. Lett. 108, 184502 (2012).

[54] O. Vincent, P. Marmottant, S.R. Gonzalez-Avila, K. Ando, C.D. Ohl, The fast dynamics of cavitation bubbles within water confined in elastic solids, Soft Matter, 10, 1455-1461 (2014)

[55] D. Mannes, W. Sonderegger, S. Hering, E. Lehmann, P. Niemz, Non-destructive determination and quantification of diffusion processes in wood by means of neutron imaging. Holzforschung 63, 589-596 (2009).

[56] W. Olek, P. Perré, J. Weres, Inverse analysis of the transient bound water diffusion in wood. Holzforschung 59, 38-45 (2005) 
[57] J. Siau, Wood: influence of moisture on physical properties. Virginia Polytechnic Institute and State University, Keene NY, USA (1995)

[58] K. Krabbenhoft, Moisture Transport in Wood - A study of Physical-Mathematical Models and their Numerical Implementation. Ph.D. thesis, Technical University of Denmark (2003)

[59] M. Goavec, S. Rodts, V. Gaudefroy, M. Coquil, E. Keita, J. Goyon, X. Chateau, P. Coussot, Strengthening and drying rate of a drying emulsion layer, Soft Matter, 14, 8612-8626 (2018)

[60] P. Faure and P. Coussot, Drying of a model soil, Physical Review E, 82, 036303 (2010)

[61] H.J. Cho, N.B. Lu, M.P. Howard, R.A. Adams, S.S. Datta, Crack formation and self-closing in shrinkable, granular packings, Soft Matter, 15, 4689-4702 (2019)

[62] N. Ben Abdelouahab, A. Gossard, S. Rodts, B. Coasne, P. Coussot, Convective drying of a porous medium with a paste cover, Eur. Phys. J. E, 5, 66 (2019)

[63] V. Bucur, Nondestructive Characterization and Imaging of Wood, Springer Series in Wood (Springer, Berlin, 2002)

[64] N.M. Holbrook, M.A. Zwieniecki, Embolism repair and xylem tension: do we need a miracle?, Plant Physiology, 120, 7-10 (1999).

[65] A.D. Stroock, V.V. Pagay, M.A. Zwieniecki, N.M. Holbrook, The physicochemical hydrodynamics of vascular plants, Annu. Rev. Fluid Mech., 46, 615-642 (2014)

[66] P. Cruiziat, T. Améglio, H. Cochard, Cavitation : a mechanism perturbating water flow in vegetals, Mec. Ind., 2, 289-298 (2001) (In French)

[67] H. Cochard, S. Delzon, Hydraulic failure repair are not routine in trees, Annals of Forest Science, 70, 659-661 (2013)

[68] C.R. Brodersen, A.J. McElrone, B. Choat, M.A. Matthews, K.A. Shackel, The dynamics of embolism repair in xylem: in vivo visualizations using High-resolution computed tomography, Plan Physiology, 154, 1088-1095 (2010)

[69] T. Knipfer, I.F. Cuneo, J.M. Earles, C. Reyes, C.R. Brodersen, A.J. McElrone, Plant Physiology, 175, 16491660 (2017) 